\title{
Towards practical autonomous deep-space navigation using X-Ray pulsar timing
}

\author{
Setnam Shemar ${ }^{1} \cdot$ George Fraser $^{2} \cdot$ Lucy Heil $^{3}$. \\ David Hindley $^{1}$ - Adrian Martindale ${ }^{2}$. \\ Philippa Molyneux ${ }^{2} \cdot$ John Pye $^{2}$. \\ Robert Warwick $^{2}$ • Andrew Lamb ${ }^{4}$
}

Received: 16 March 2016 / Accepted: 1 April 2016 /Published online: 30 July 2016

(C) The Author(s) 2016. This article is published with open access at Springerlink.com

\begin{abstract}
We investigate the feasibility of deep-space navigation using the highly stable periodic signals from X-ray pulsars in combination with dedicated instrumentation on the spacecraft: a technique often referred to as ' $\mathrm{XNAV}$ '. The results presented are based on the outputs from a study undertaken for the European Space Agency. The potential advantages of this technique include increased spacecraft autonomy and lower mission operating costs. Estimations of navigation uncertainties have been obtained using simulations of different pulsar combinations and navigation strategies. We find that the pulsar PSR B1937+21 has potential to allow spacecraft positioning uncertainties of $\sim 2$ and $\sim 5 \mathrm{~km}$ in the direction of the pulsar after observation times of 10 and $1 \mathrm{~h}$ respectively, for ranges up to $30 \mathrm{AU}$. This could be achieved autonomously on the spacecraft using a focussing X-ray instrument of effective area $\sim 50 \mathrm{~cm}^{2}$ together with a high performance atomic clock. The Mercury Imaging X-ray Spectrometer (MIXS) instrument, due to be launched on the ESA/JAXA BepiColombo mission to Mercury in 2018, is an example of an instrument that may be
\end{abstract}

\section{GF deceased 18 March 2014}

Setnam Shemar

setnam.shemar@npl.co.uk

$\triangle$ John Pye

pye@leicester.ac.uk

1 National Physical Laboratory, Hampton Road, Teddington, Middlesex TW11 0LW, UK

2 Department of Physics and Astronomy, University of Leicester, University Road, Leicester LE1 7RH, UK

3 Anton Pannekoek Institute for Astronomy, University of Amsterdam, Postbus 94249, Amsterdam, GE 1090, The Netherlands

4 UK National Quantum Technology Hub in Sensors and Metrology, School of Physics and Astronomy, University of Birmingham, Birmingham B15 2TT, UK 
further developed as a practical telescope for XNAV. For a manned mission to Mars, where an XNAV system could provide valuable redundancy, observations of the three pulsars PSR B1937 + 21, B1821-24 and J0437-4715 would enable a three-dimensional positioning uncertainty of $\sim 30 \mathrm{~km}$ for up to 3 months without the need to contact Earth-based systems. A lower uncertainty may be achieved, for example, by use of extended observations or, if feasible, by use of a larger instrument. X-ray instrumentation suitable for use in an operational XNAV subsystem must be designed to require only modest resources, especially in terms of size, mass and power. A system with a focussing optic is required in order to reduce the sky and particle background against which the source must be measured. We examine possible options for future developments in terms of simpler, lower-cost Kirkpatrick-Baez optics. We also discuss the principal design and development challenges that must be addressed in order to realise an operational XNAV system.

Keywords XNAV·Spacecraft $\cdot$ Navigation $\cdot$ Pulsar $\cdot$ X-ray $\cdot$ Timing

\section{Introduction}

The navigation of deep space missions is currently achieved by the ESA's European Space Tracking (ESTRACK) network and NASA's Deep Space Network (DSN) [18]. Each of these employ a global network of large ground-based radio antennas. In recent years space agencies around the world have been exploring the feasibility of using Xray pulsars for spacecraft navigation. Whereas the DSN and ESTRACK networks require a spacecraft to communicate with ground-based systems, the use of pulsars would to some extent enable autonomous navigation on-board the spacecraft thereby minimising communications with Earth. This would also offer the potential of lower mission operating costs due to the reduced need for ground infrastucture.

Pulsars are ultra-compact, rapidly rotating and strongly magnetised neutron stars that are the remnants of the death of a massive star in a supernova explosion [50]. The high timing stability of the pulses from certain types of pulsars has led to important developments including strong evidence for the existence of gravitational waves [80]. It was first speculated that pulsars could be used for time and position determination for spacecraft by Reichley et al. [63] and Downs [22]. Chester and Butman [14] described the potential use of X-ray pulsars for spacecraft navigation due to the much smaller detector size required compared to that for radio pulsars (see also review by [8]).

The main objective of the work presented here was to investigate the feasibility of deep space navigation using XNAV based on X-ray pulsars from a high-level perspective considering also the X-ray instrumentation. Section 2 provides a brief review of literature on pulsar-based navigation concepts relevant to this study. In Section 3 we present a catalogue of X-ray pulsars that are potentially suitable for the navigation concepts and some of their relevant characteristics and parameters. Section 4 analyses the potential Position, Velocity and Time (PVT) estimation errors that can be obtained using different combinations of X-ray pulsars and navigation strategies. In Section 5 we describe the X-ray instrumentation technology that is currently available for use in an $\mathrm{XNAV}$ system as well as potential future developments. Section 6 discusses the performance and limitations of an XNAV system and compares these to those of the ESTRACK and DSN networks. Finally, in Section 7 we give the conclusions. 


\section{Relevant concepts for navigation}

This technique is based on ranging and velocity estimation by use of pulsar timing and frequency measurements. For a given pulsar, the time interval between pulses is referred to as the pulse period, $P$, and this generally changes slowly and uniformly with time. The pulse periods of different pulsars can range between $1.5 \mathrm{~ms}$ and several seconds. Here we firstly describe the measurement of pulse Times-Of-Arrival (TOAs, see e.g. [48], Section 8.1) and the processing necessary for such a system together with the pulsar timing models. We then focus on navigation strategies suitable for PVT estimation.

We briefly review here the main aspects directly relevant to our study; the reader is referred to the published literature (e.g. [34, 69]) for a more extensive view. In particular, we restrict ourselves to consideration of two navigation methods (utilising one pulsar and three or four pulsars) and the periodic signal from rotation-powered Xray pulsars.

\subsection{Pulse timing}

One of the most important requirements for such a system is for the spacecraft to have access to a reliable timing model for the pulsar. The most accurate models are often derived from radio observations on Earth. The pulse phase of a pulsar can be modelled and specified at a known location, for example the Solar System Barycentre (SSB), using the measured pulse phase and pulse frequency of the pulsar at a given epoch [48, $50,69]$. The model will not be perfect since the exact values of pulse phase, pulse frequency and derivatives will not be known and the TOAs will be affected by measurement error. Furthermore, some pulsars can also exhibit significant timing irregularities called 'timing noise' (e.g. [39]) and 'glitches' (e.g. [25, 71]) which are intrinsic to the rotation of a star.

An X-ray instrument on-board the spacecraft is used to obtain time-resolved measurements of a given pulsar. Pulse TOAs are then extracted by comparing the measured time-series with a model pulse profile [24, 31, 36, 68]. Figure 1 (see also Section 2.2.1) shows lines representing a given pulse phase of a pulsar signal arriving at the true spacecraft position and an initial estimate of the position at two instants in time, separated by an interval $\Delta t$, as the signal moves through the Solar System relative to the inertial reference frame of the SSB [69]. In the simplest case, the pulse TOAs measured at the spacecraft are compared with those predicted at the SSB by the timing model in order to obtain a corrected spacecraft position estimate along the direction of the pulsar. This requires conversion of the measured TOAs to Barycentric TOAs, using the initial position estimate and the unit vector to the pulsar, $\underline{\hat{n}}$, with respect to the SSB [69]. These are the key pulsar timing elements that enable positioning of the spacecraft in the direction of a given pulsar.

\subsection{Navigation}

Here we describe some navigation strategies suitable for PVT estimation. These can be categorized as delta-correction and absolute navigation techniques [34] and will be discussed in turn below. In all cases it is necessary that the pulsar timing models for 


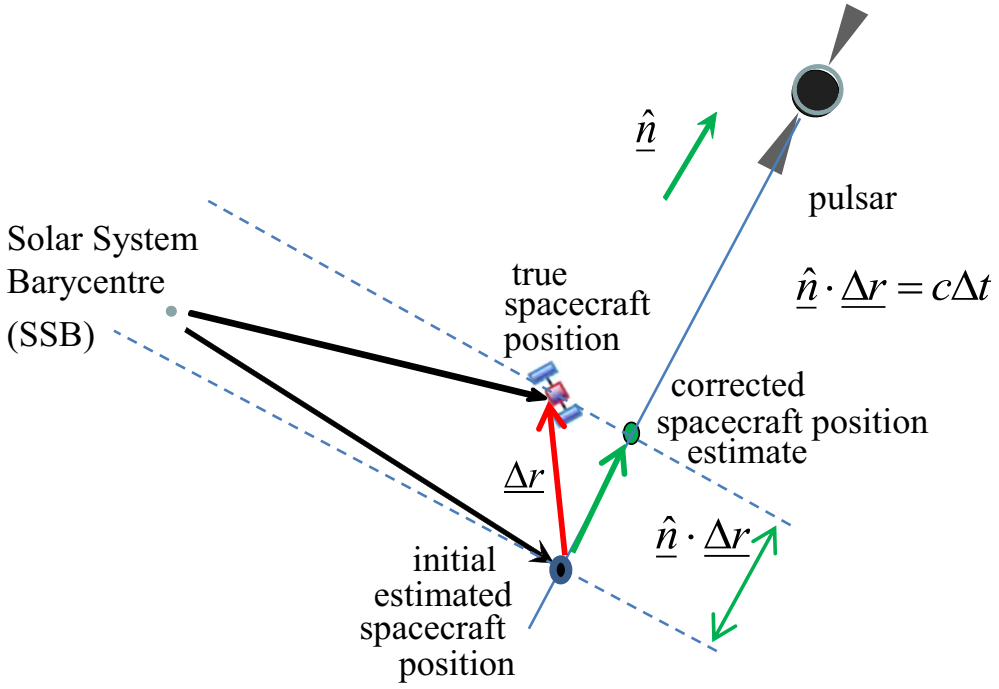

Fig. 1 A simplified approach for measuring the position of a spacecraft in the direction of a pulsar. The dashed lines represent a given pulse phase of a signal from the pulsar arriving at the true spacecraft position and an initial estimated position at two instants in time separated by an interval $\Delta t$. The 'delta-correction' is shown as $\underline{\hat{n}} \cdot \underline{\Delta r}$ and is equal to $c \Delta t$, where $c$ is the speed of light. The green point represents the corrected spacecraft position along the direction of the pulsar

relevant pulsars are updated sufficiently frequently within the XNAV processing system such that the errors arising from these do not contribute significantly to the navigation uncertainties. These updates would be carried out for example using the ESTRACK or DSN networks, whilst the interval between updates would depend on the pulsars employed, but could vary for example between one update per day to a year.

\subsubsection{Delta-correction measurement using a single pulsar}

This is the simplest strategy and represented in Fig. 1. A more detailed description of this can be found in $[34,69]$. An initial estimate of the spacecraft position is required to within $c P / 2$, where $c$ is the speed of light, as well as an estimate of the velocity. These might be obtained using for example the DSN, ESTRACK or an orbit propagation process within the spacecraft navigation system [69]. An accurate time reference onboard the spacecraft enables measurement of the observed pulse TOAs from a single pulsar. If the initial spacecraft position estimate used to convert the TOAs to the SSB is correct and there are no TOA measurement errors then there will be no time-offset, $\Delta t$, compared to the TOAs predicted by the pulsar's timing model. However, if the initial position estimate is in error then a non-zero time-offset, $\Delta t$, will be measured corresponding to a position-offset in the direction of the pulsar, $\underline{\hat{n}} \cdot \underline{\Delta r}=c \Delta t$, where $\underline{\hat{n}}$ is the unit vector to the pulsar with respect to the SSB and $\underline{\Delta r}$ is the error in the initial position estimate [69]. This position-offset is often referred to as the 'delta-correction' and enables a corrected spacecraft position estimate along the direction of the pulsar to be obtained. This strategy is relatively simple to implement as it requires an X-ray instrument that observes only a single pulsar at a time. It can, in principle, be extended to sequential observations of multiple pulsars to obtain two- or three-dimensional 
position estimates although it would depend on the motion of the spacecraft and being able to adequately take account of this [69].

\subsubsection{Absolute navigation}

This strategy offers the potential for a spacecraft to autonomously determine its absolute position in three dimensions with respect to an inertial reference frame [69, 70]. The advantage of this is that it can navigate and restart without the aid of another method such as DSN. As shown in Fig. 2, by using measurements of pulse phase from four or more sources it would also be possible to measure and correct for any spacecraft clock time-offset. Alternatively three pulsars could be used if a high-accuracy time reference is available on-board the spacecraft, for example using a high-performance atomic clock. A difficulty arises because a pulsar signal does not include information to identify the number of the particular pulse received. This results in pulse or cycle ambiguities, a problem that can be overcome by combining the measured pulse phase information of several pulsars and using knowledge of the unit vector of each in order to identify the unique set of cycles that satisfies the measurements [69]. A priori knowledge such as an approximate location of the spacecraft within the Solar System and an estimate of the spacecraft velocity together with observations of longer period pulsars may initially be required in order to reduce the parameter search space [68].

Absolute navigation would in principle be the most versatile for many types of space missions depending on its performance. A disadvantage is that it requires simultaneous observations of multiple pulsars which would require multiple detectors, making this strategy more difficult to implement. Whether or not it would be possible to use data taken sequentially for different pulsars using a single detector would depend on the motion of the spacecraft and being able to adequately take account of this (Deng et al.

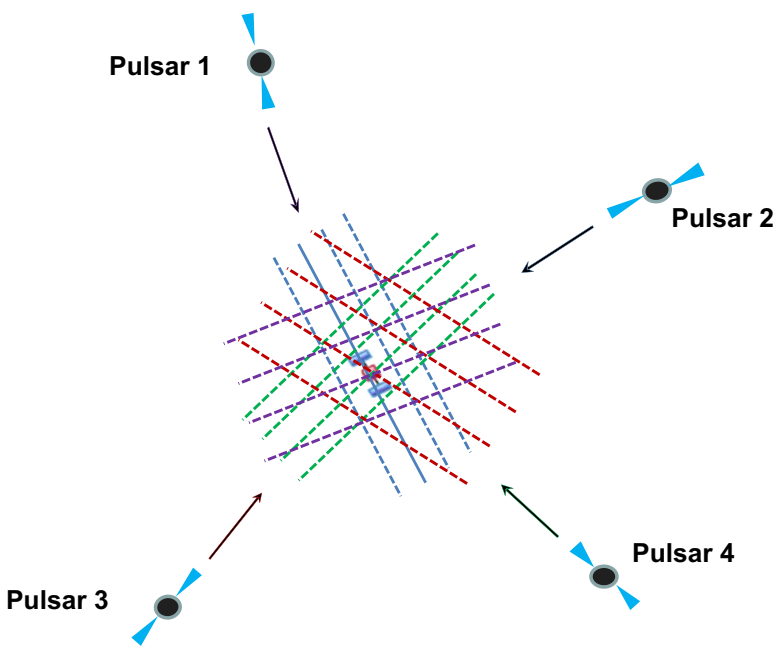

Fig. 2 Absolute navigation using simultaneous observations of a minimum of four pulsars enabling measurement of the spacecraft three-dimensional position $(x, y, z)$ and the on-board clock time-offset, $t_{c}$, from terrestrial time scales. The dashed lines represent candidate lines of position for each pulsar separated by $c P$ in each case and obtained using the measured pulse phases of the four pulsars at a given time 
[21]). The noise in the spacecraft clock is unlikely to significantly degrade the quality of such sequential observations spanning over periods of $1 \mathrm{~h}$ to 1 day. This is because a relatively modest performance oscillator with a stability of $10^{-12}$ for averaging times of 1 day will lead to a range measurement error contribution of $30 \mathrm{~m}$ which is small compared to other error sources. An atomic oscillator of such a performance should be readily achievable on a spacecraft. Furthermore, with careful design, a quartz-based oscillator could be expected to exhibit a similar performance [11].

\subsubsection{Determining velocity}

An accurate approach for spacecraft velocity measurement involves comparing the frequency, or the rate, of pulses received at the spacecraft from one or more pulsars with those predicted at the SSB [69]. Any relative velocity between the pulsar and spacecraft will result in the interval between the received pulses either decreasing or increasing due to the Doppler Shift. After correcting for any motion of the pulsar relative to the $\mathrm{SSB}$, any Doppler shift observed in the pulse timing has to be due to the motion of the spacecraft in the direction of the pulsar. By taking measurements from multiple pulsars it is possible to obtain velocity components of the spacecraft in three dimensions.

\section{X-ray pulsars and their characteristics}

We present the available X-ray pulsars and their characteristics, including their skyposition (i.e. astrometric) uncertainties. We also describe a simple analytical approach to estimate spacecraft range error, parameterised in terms of the pulsar properties and $\mathrm{X}$-ray measurements, and compare this with results from simulations of detailed pulse profiles.

\subsection{Compiling an X-ray pulsar catalogue for XNAV}

There are over 2000 known pulsars, most of which were discovered in the radio band [52]. Approximately 100 of these are Rotation-Powered X-Ray Pulsars (RP-XRPs) [7], with around 35 of these having been detected with pulsed X-ray emission and having a measured pulse profile, as reported in the literature to $\sim$ mid-2012 - the 'census epoch' of our study. These XRPs, which have rotation periods ranging from $\sim 1.5$ to $100 \mathrm{~s}$ of milliseconds [7], are the main focus of this work. Accretion-powered pulsars (see e.g. [7]), are rather poor in terms of pulse stability (and some are unsuitable as they are highly transient in flux, and some have rather long periods, of seconds or greater), though they may have some utility provided there are associated X-ray monitoring observations from a low-Earth orbit satellite.

The majority of the rotation-powered pulsar population are relatively bright and young with ages $\sim 10^{3}$ to $\sim 10^{7} \mathrm{yr}$ [7]; the most famous example of which is the Crab pulsar (PSR B0531+21), born in AD 1054. A relatively small fraction can exhibit extremely high long-term timing stability - excellent attributes for a 'celestial clock' [10, 24, 69]. These are the 'MilliSecond Pulsars' (MSPs) which have the shortest periods and are relatively weak in strength. They are relatively old objects with ages of typically $\sim 10^{7}$ to $\sim 10^{10} \mathrm{yr}$ (see [7], Fig. 6.2). However, young pulsars such as the Crab 
may also be useful if monitored sufficiently frequently from the ground (in radio) or from an X-ray monitoring satellite. Of order $10 \%$ of all known pulsars are MSPs; however, they constitute $\sim 50 \%$ of known rotation-powered X-ray pulsars, and $\sim 30 \%$ of those with detected pulsed emission in X-rays [7].

With the exception of the Crab pulsar which is one of the brightest X-ray sources in the sky, other known rotation-powered pulsars have X-ray fluxes measured at the Earth typically a thousand times fainter. Even then only a small number of sources $(\sim 3)$ are detected at the 'milli-Crab' level, with the rest being at least a factor of 5-10 fainter still.

General X-ray source catalogues such as those from ROSAT [76, 77], XMMNewton [78] and Chandra [26] are available for pulsar X-ray source fluxes, whilst general astronomical object catalogues such as $\mathrm{CDS} \mathrm{SIMBAD}^{1}$ provide information on general source properties and categorisation. Furthermore, the ATNF pulsar 'master' catalogue $^{2}$ [52] provides detailed pulsar ephemerides and celestial coordinates.

The starting point for generation of a catalogue of relevant properties of RP-XRPs were the tabulations in Becker's [7] review (these list 89 objects), together with the other databases mentioned above. These were augmented and updated from published literature (e.g. $[30,66])$; in particular, the X-ray pulse width and fractional pulsed signal had to be extracted for each individual pulsar. A catalogue was compiled comprising data on 89 RPXRPs, with the most detailed information being provided for the 35 RP-XRPs for which pulsed X-ray emission profiles were found in the literature. Table 1 lists some parameters for the 35 RP-XRPs with detected pulsations, including source flux, ${ }^{3}$ fractional pulse width, astrometric position error and estimates of the spacecraft range error contributions for 'focussing' and 'collimated' instrument types. The objects are ordered by increasing range error for a focussing instrument. The given range errors represent 1-sigma and are computed according to the analytic formula in Eq. A.3 (see also Section 3.3.1) using an observation time, $\mathrm{T}_{\mathrm{obs}}=5 \times 10^{3} \mathrm{~s}$, and instrument effective area, $\mathrm{A}_{\mathrm{eff}}=0.005 \mathrm{~m}^{2}$, according to an 'improved Point Spread Function (PSF)' version of the BepiColombo Mercury Imaging X-ray Spectrometer (MIXS) instrument as described in Section 5. This corresponds to a time-area product of $250 \mathrm{~m}^{2} \mathrm{~s}$. As can be seen from Eq. A.3, the range error contribution due to the instrument is inversely proportional to the square root of the time-area product. Figure 3 gives a plot of the ecliptic longitude versus ecliptic latitude of each of the 35 pulsars to show the distribution of the pulsars on the sky.

\subsection{Pulsar position uncertainties}

The current status of pulsar position ${ }^{4}$ uncertainties is summarised in Table 1, column 'Position error', using the data from the ATNF catalogue [52], supplemented by an

\footnotetext{
$\overline{{ }^{1} \text { http://simbad.u-strasbg.fr/simbad/ }}$

${ }^{2} \mathrm{http} / / / \mathrm{www}$. atnf.csiro.au/people/pulsar/psrcat/

${ }^{3} 26$ of the sources had XMM fluxes available; these were all utilised. The origins of the fluxes for the remaining sources, and the corresponding numbers of sources, were: Chandra -1 , ROSAT PSPC -3 , ROSAT HRI - 3, ASCA - 1, BeppoSax - 1. This ranking also indicates the preference order for selecting fluxes. Where ROSAT count rates were used they were converted to fluxes using a factor $1.24 \times 10^{-11}$ (PSPC) and $3.66 \times 10^{-11}$ (HRI) erg cm ${ }^{-2} \mathrm{~s}^{-1} /$ count/s $(0.1-2.4 \mathrm{keV})$. To convert all 'energy' fluxes to photon $\mathrm{cm}^{-2} \mathrm{~s}^{-1}$, we have used a factor $4 \times 10^{8} \mathrm{ph} / \mathrm{erg}$. We have not attempted to take account of the spectral shapes of individual sources.

${ }^{4}$ 'Position' here means the astrometric location of the pulsar on the sky, in any 'sky' coordinate frame e.g. equatorial (RA, Dec), ecliptic, galactic.
} 


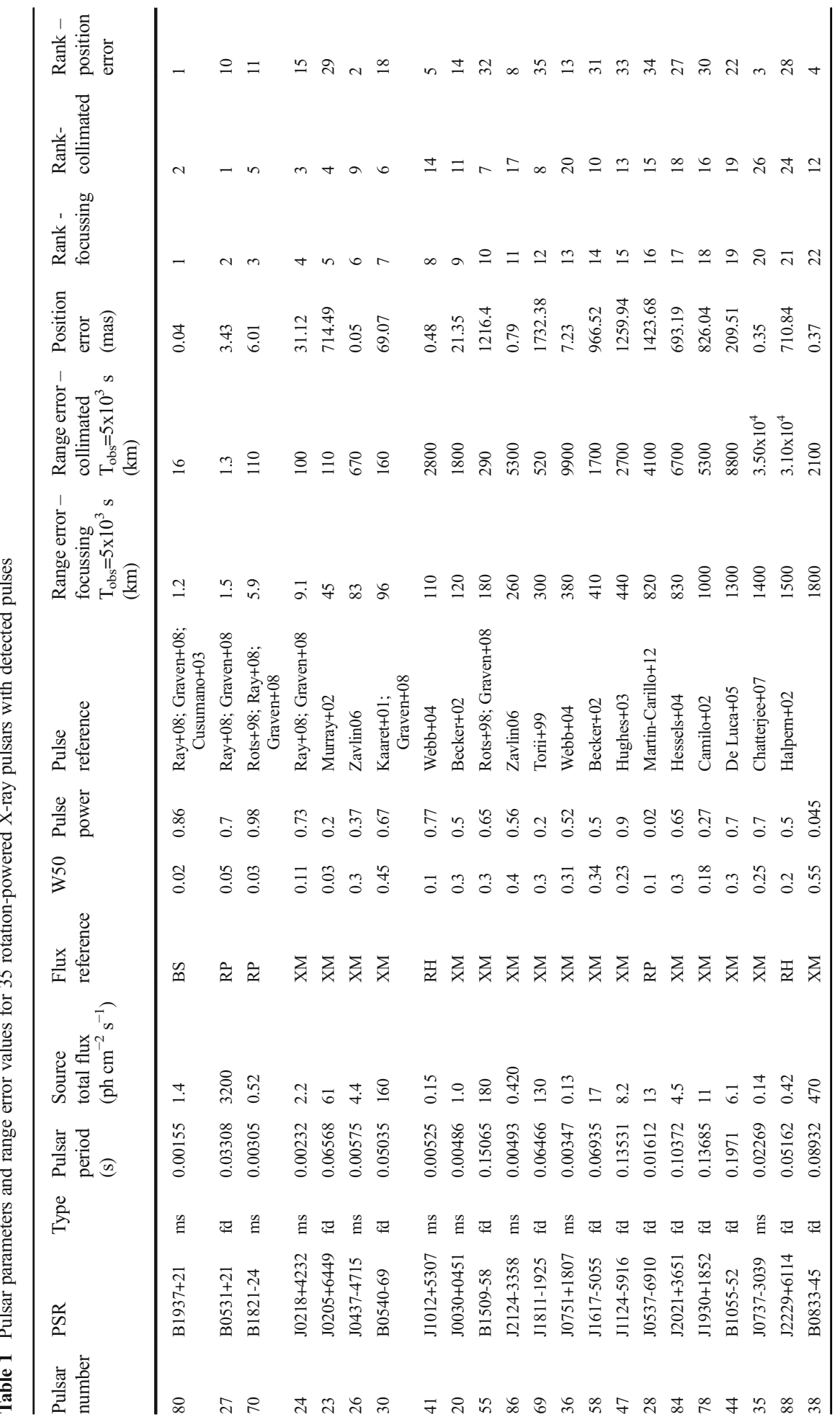




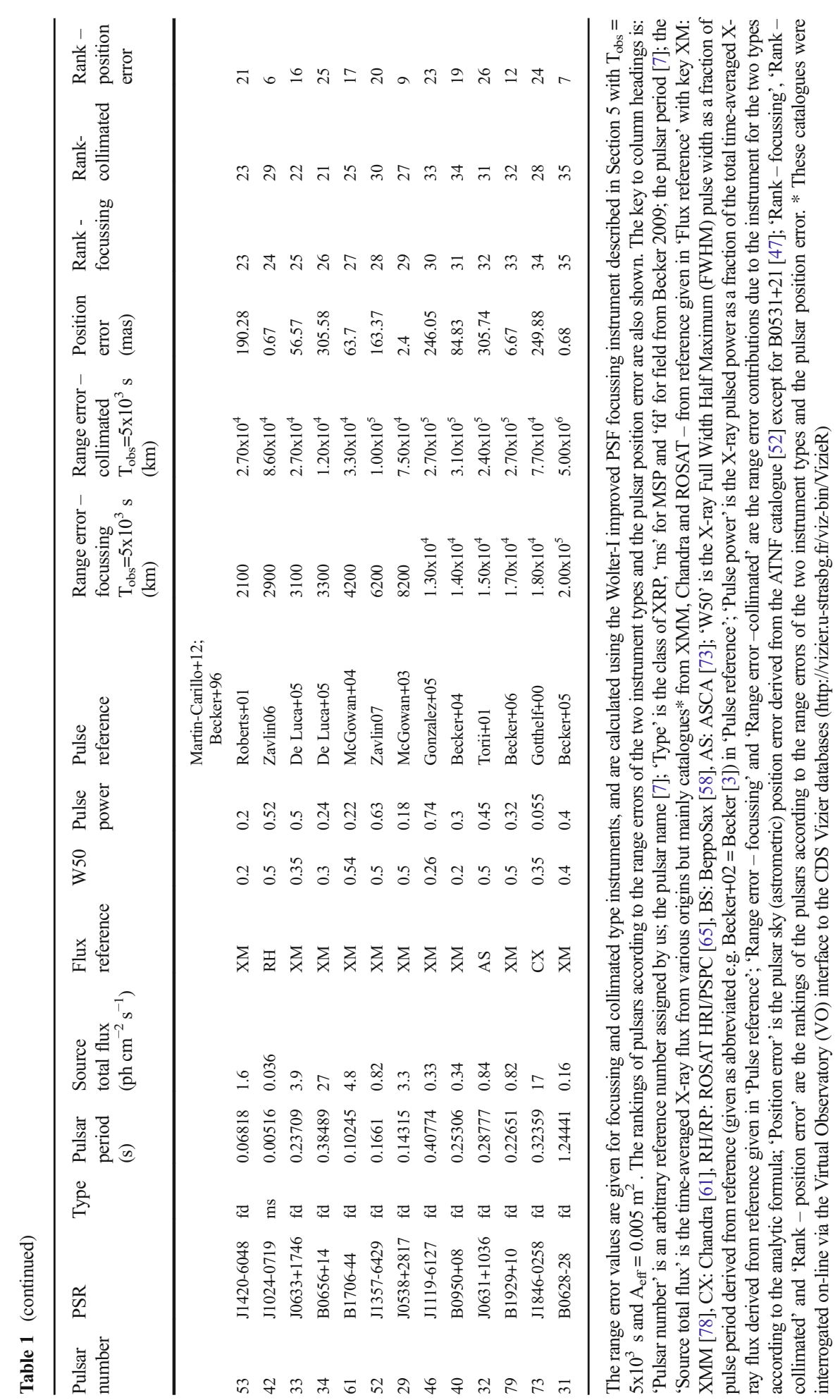




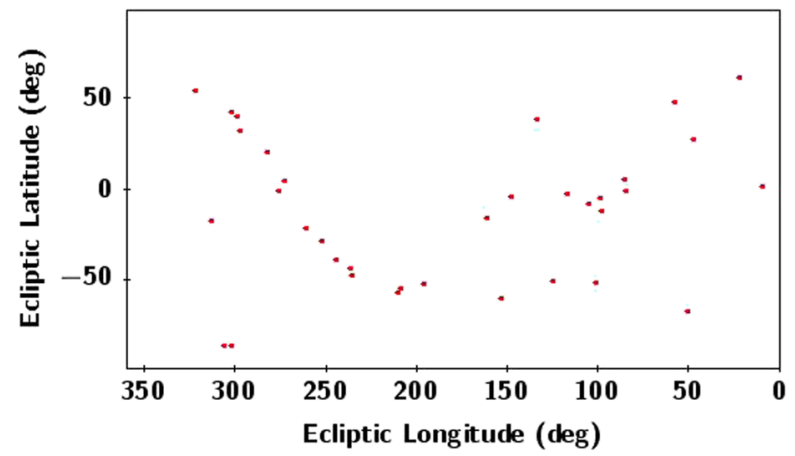

Fig.3 Sky distribution in ecliptic coordinates of 35 X-ray pulsars relevant to this study, as listed in Table 1

improved value for the Crab pulsar [47]. Of the 35 pulsars listed, there are 8 with an overall position uncertainty of $<1$ mas, and 2 of these have a position uncertainty of $<0.1$ mas. Astrometry using X-ray, optical or 'standard' (e.g. relatively short baseline) radio measurements typically yields position uncertainties of $\sim 0.1-1$ arcsec. Higher precision astrometry for pulsars generally requires either pulse timing techniques or Very Long Baseline Interferometry (VLBI) (see e.g. [47, 48]). Long-term programmes to obtain high-precision pulsar positions and timing are in progress, driven by goals such as detection of gravitational-waves (see e.g. [38]). These programmes, and new instrumentation, e.g. the Square Kilometre Array (SKA) and its precursors, should lead to improved position uncertainties. It should also be noted that for high-precision positions, the effects of proper motion will need to be taken into account (see e.g. [48]) and possibly updated with contemporaneous measurements during XNAV operations.

\subsection{Estimation of range error contributions due to the instrument}

\subsubsection{Using an analytic formula}

A simple analytic formula can be used to estimate the spacecraft range measurement error based on the TOA measurement uncertainty alone by using the characteristics of each pulsar and the proposed instrumentation (e.g. [62]). This can be used for both focussing and collimated instrument types depending upon the input parameters and is largely independent of the specific method of TOA determination, such as crosscorrelation, least-squares fitting or maximum likelihood. The formula and relevant details are given in Appendix A.

The above approach enables us to generate a ranking for the objects according to likely utility for XNAV, albeit with some simplifications and limitations. These include ignoring the detailed shape of the pulse profile and any variation with photon energy of the pulse profile and fractional pulsed emission; such energy dependence is evident in some cases, and differs in form between objects. In addition, some XRPs such as the Crab and Vela pulsars lie within a region of significant extended X-ray emission due to the associated supernova remnant or a pulsar-wind nebula. We have attempted, where possible, to quote relatively low values for the pulsed fraction in such cases, recognising that a feasible XNAV instrument may not have sufficient spatial resolution to 'reject' the extended emission, which thus forms an additional background to the desired pulse signal. Figure 4 


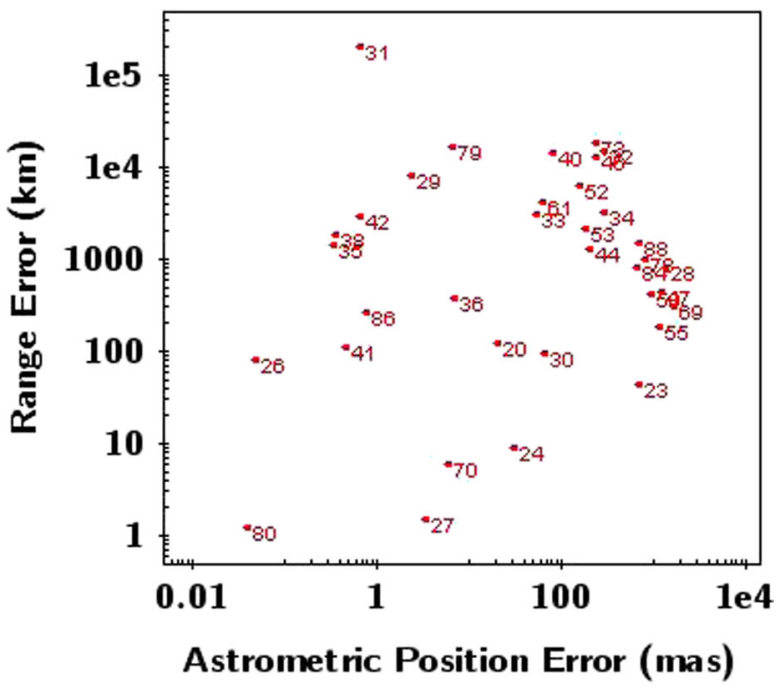

Fig. 4 Estimates of spacecraft range errors obtained using the analytic formula for 35 rotation-powered X-ray pulsars observed using the improved PSF focussing instrument described in Section 5 with $\mathrm{T}_{\text {obs }}=5 \times 10^{3} \mathrm{~s}$ and $\mathrm{A}_{\mathrm{eff}}=0.005 \mathrm{~m}^{2}$, versus pulsar astrometric position error. The most suitable sources for XNAV are thus towards the lower left of the plot. Each pulsar is identified by its pulsar number from Table 1, column 1; thus the 5 pulsars discussed in detail in this paper are numbers 26 (J0437-4715), $27(\mathrm{~B} 0531+21), 41(\mathrm{~J} 1012+5307), 70$ (B1821-24) and $80($ B1937+21)

shows the range-error estimates for a focussing instrument, with $\mathrm{T}_{\mathrm{obs}}=5 \times 10^{3} \mathrm{~s}$, versus astrometric position error. The values are also listed in Table 1.

The scaling of the range errors (or TOA errors) for the different instrument types with observation time and effective area, and ranking of the pulsars according to these values, are relatively insensitive to the precise choice of background values. For example, assuming zero background does not change the set of 10 pulsars with the lowest range errors for the focussing instrument, though there are some changes in the ranking, and leads to a change in two cases near the lower boundary, for the collimated instrument.

\subsubsection{Simulation of pulse profiles and comparison of range error estimates}

We have performed simulations to examine in detail the effects of specific pulse profiles and instrument characteristics on the achievable TOA and range errors. The calculations used a model of the pulsed and unpulsed signal from the pulsar and the background fluxes, folded through the assumed instrument response and for a specified integration time, and with Poisson noise incorporated. Each simulated dataset was cross-correlated with the noiseless, model profile to determine a TOA error; the simulation was repeated typically 1000 times for each 'observation' in order to build-up a TOA-error distribution. An example, based on PSR $\mathrm{B} 1937+21$ as if viewed by our 'baseline' focussing instrument for an integration time of $50 \mathrm{ks}$, is shown in Fig. 5. The range error obtained from the simulations is computed as $\mathrm{W}_{68} / 2$, where $\mathrm{W}_{68}$ is the width of the distribution between the $16 \%$ and $84 \%$ percentiles (enclosing $68 \%$ of the area of the distribution equivalent to the 1-sigma for a Gaussian distribution). This is a somewhat more 'robust' 

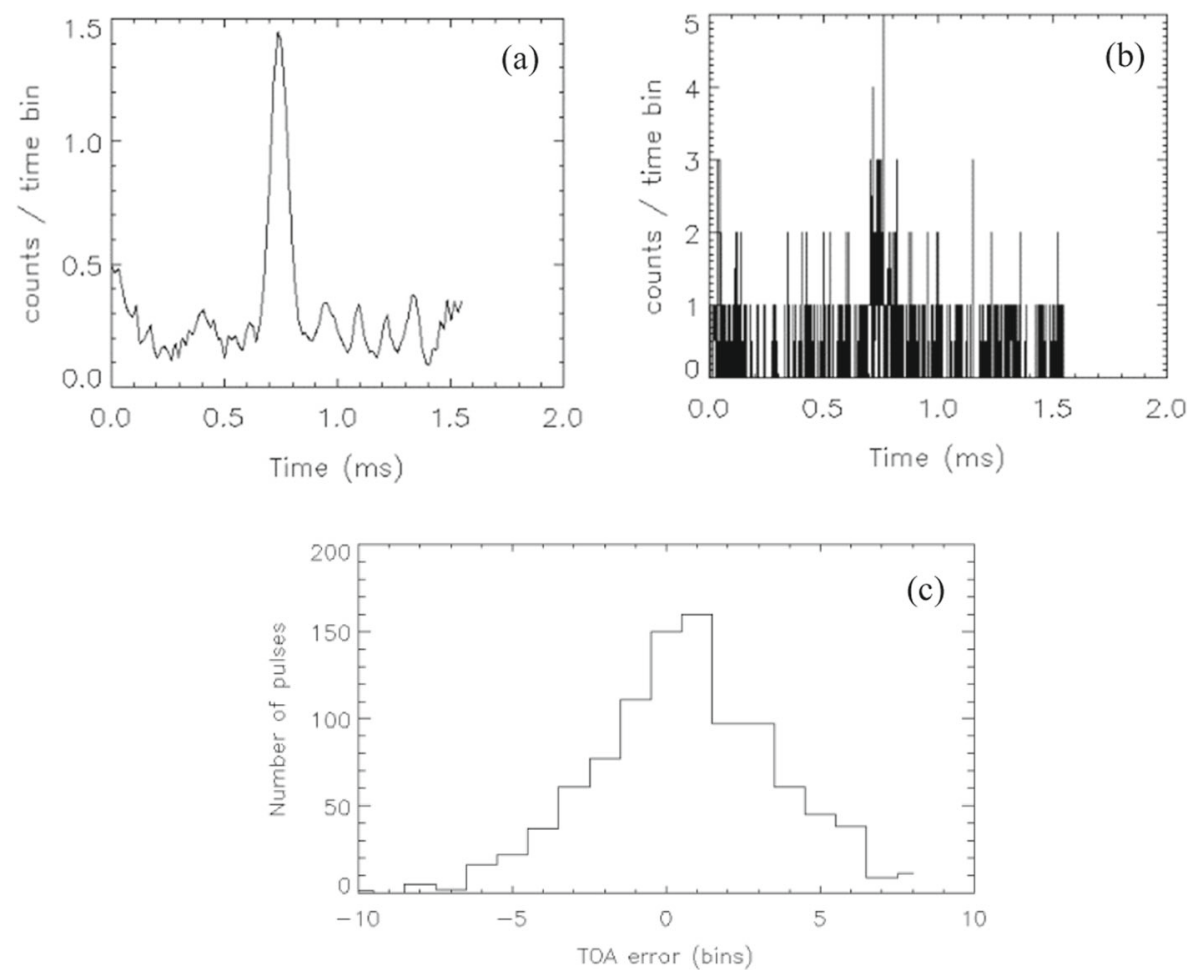

Fig. 5 An example of pulse TOA simulations for PSR B1937+21 using an integration time of $5 \times 10^{4} \mathrm{~s}$ with a BepiColombo-MIXS-type instrument. The plots show as follows: a the input (noiseless) model pulse profile based on data from the ROSSI X-ray Timing Explorer (RXTE), b the simulated pulse profile summed over the duration of the observation and $\mathbf{c}$ the TOA error distribution resulting from the simulated data where 1 bin $\approx$ $1.55 \mu$ s i.e. $10^{-3}$ of the pulse period

estimator than directly computing the standard deviation, given the 'noise wings' present for low SNR cases.

More accurate and complex formulae can be derived by detailed consideration of pulse shapes and statistics (e.g. [24, 36]); however the analytic formula described in Section 3.3.1 and Appendix A serves to highlight the main parametric dependencies. For a wide range of pulse-profile shapes and SNR, the values given by the formula and determined by the simulations for the five pulsars PSR B1937+21, B1821-24, J0437-4715, J1012 + 5307 and B0531 + 21 agree to within a factor of 3 , and scale approximately with $1 / \sqrt{ } \mathrm{T}_{\mathrm{obs}}$ as expected and as can be seen in Table 2 . A qualitative indication of the pulse shape in each case is also given in Table 2. We can thus have confidence in taking the analytic formula as an approximate guide, useful for indicating functional dependencies on the various parameters and for ranking the pulsars in terms of range error, whilst the simulations allow a more detailed evaluation of specific cases, for example the effects of low SNR or complex pulse profiles.

Comparison of our range-error estimates with some of those reported elsewhere [24, $34,62]$, and bearing in mind possible differences in assumptions such as for pulsed and unpulsed fluxes and background fluxes, indicates general consistency. 


\section{Estimation of navigation and timing uncertainties from simulations}

Two navigation strategies were outlined in Section 2; delta-correction using a single pulsar and absolute navigation using three or four pulsars. Appendix B describes the Monte Carlo approaches used to simulate the navigation errors achievable by each of these and some of the simulation outputs. These are used to identify the X-ray pulsars that would provide the lowest navigation uncertainties for each strategy. Here we present uncertainty budgets for PVT estimation using the best-performing pulsars identified for each navigation strategy. Finally, we summarise the results of the total uncertainties for PVT estimation in each case.

\subsection{Navigation and timing uncertainty budgets}

Simulations of errors are carried out using a similar approach to that in [34] (see Appendix B) using propagation of errors in the small perturbation case. These are partly based on the range errors due to the X-ray instrument for observation times, $\mathrm{T}_{\mathrm{obs}}=5 \times 10^{3}$ and $5 \times 10^{4} \mathrm{~s}$, obtained using the analytic formula (see Section 3.3.1). For improved uncertainty estimates, range errors obtained from simulations (see Section 3.3.2) instead of the analytic formula have been used for the five pulsars PSR B1937+21, B1821-24, J0437-4715, J1012 + 5307 and B0531+21. In the case of the latter pulsar, the errors are obtained by appropriate scaling of the values given in Table 2.

For the results presented in this section, and as described in Appendix B, a latency period of 3 months is assumed in the cases of four pulsars. This refers to the interval between two timing model updates during a period of autonomous navigation and allows us to take account of the range error contributed by pulsar timing model error. In the case of the Crab pulsar, a much shorter period of order 3 days has been assumed.

Table 2 Comparison of range-error estimates from the analytic formula and simulations

\begin{tabular}{|c|c|c|c|c|c|c|}
\hline PSR & $\begin{array}{l}\text { Pulse Period } \\
(\mathrm{ms})\end{array}$ & $\begin{array}{l}\text { Pulse fractional } \\
\text { width, FWHM }\end{array}$ & Pulse shape & $\begin{array}{l}\text { Observation } \\
\text { time, } \mathrm{T}_{\mathrm{obs}}(\mathrm{ks})\end{array}$ & $\begin{array}{l}\text { Range error } \\
(\mathrm{km}) \text {, from } \\
\text { formula }\end{array}$ & $\begin{array}{l}\text { Range error } \\
(\mathrm{km}) \text {, from } \\
\text { simulation }\end{array}$ \\
\hline \multirow[t]{2}{*}{ B1937+21 } & 1.55 & 0.02 & sharp, single & 5 & 1.2 & 4.4 \\
\hline & & & & 50 & 0.38 & 1.2 \\
\hline \multirow[t]{2}{*}{ B1821-24 } & 3.05 & 0.03 & sharp, double & 5 & 5.9 & 10.0 \\
\hline & & & & 50 & 1.9 & 2.8 \\
\hline \multirow[t]{2}{*}{ J0437-4715 } & 5.75 & 0.3 & $\begin{array}{l}\text { broad, asymmetric } \\
\text { single }\end{array}$ & 5 & 83.0 & 67.0 \\
\hline & & & & 50 & 26.0 & 16.0 \\
\hline \multirow[t]{2}{*}{$\mathrm{J} 1012+5307$} & 5.25 & 0.1 & sharp, single & 5 & 110.0 & 41.0 \\
\hline & & & & 50 & 35.0 & 23.0 \\
\hline \multirow{2}{*}{$\begin{array}{l}\text { B0531+21 } \\
\quad(\text { Crab })\end{array}$} & 33.08 & 0.05 & sharp, double & 0.01 & 34.0 & 55.0 \\
\hline & & & & 0.1 & 10.5 & 15.0 \\
\hline
\end{tabular}

The results are based on the Wolter-I design with improved PSF described in Section 5 
As the errors used as input to the simulations are representative of 1-sigma uncertainties, the outputs of the simulations are equivalent to 1-sigma uncertainties. These should be taken as indicative values because they vary depending on the exact values of the input data used for each pulsar.

The outputs are expressed in the form of uncertainty budgets for the best performing and other selected pulsars identified for each navigation strategy. These apply specifically to a spacecraft located in the ecliptic plane at a distance of $30 \mathrm{AU}$ from the SSB in the direction of zero degrees ecliptic longitude and latitude, corresponding to ecliptic coordinates given by $x=30 \mathrm{AU}, y=0 \mathrm{AU}$ and $z=0 \mathrm{AU}$. The velocity vector is taken to be of magnitude $30 \mathrm{kms}^{-1}$ in the direction of zero degrees ecliptic longitude and latitude. However, in most cases the position uncertainty budgets are found to be reasonably representative of spacecraft ranges of $30 \mathrm{AU}$ in other directions from the SSB within the ecliptic plane. The largest variation is found in the single pulsar case of using the Crab pulsar. Here, for example, the position uncertainty budget can be lower by about one order of magnitude in the direction of $90^{\circ}$ ecliptic longitude and zero degrees latitude, corresponding to ecliptic coordinates given by $x=0 \mathrm{AU}, y=30 \mathrm{AU}$ and $z=0$ AU. The simulations do not take account of any knowledge about the trajectory of the spacecraft and the use of a Kalman filter.

The navigation and timing uncertainty budgets comprise mainly two components (see Eqs B.2 to B.10 in Appendix B). The first is largely due to the TOA measurement uncertainties arising from the instrument, with a contribution also from the pulsar timing model uncertainty. The second is due to the pulsar position uncertainties on the sky, which leads to the spacecraft position and clock time-offset uncertainties increasing linearly with range from the SSB, whilst the velocity and time-drift rate uncertainties increase linearly with velocity.

Table 1 demonstrates that a higher performance is expected from a focussing instrument and consequently the results presented in this section are based on using this type of instrument. The effective area is based on existing technology, i.e. the BepiColombo MIXS-T instrument [29] which has an effective area of $0.005 \mathrm{~m}^{2}$, as described in Section 5; we assume an improved PSF, as also described in Section 5.

Table 3 presents the uncertainty budgets with the two components for the three pulsar-set PSR B1937+21, B1821-24 and J0437-4715 which gives the lowest position uncertainty for $\mathrm{T}_{\mathrm{obs}}=5 \times 10^{4} \mathrm{~s}$ and for PSR B1937+21, B0531+21 and J0437-4715 which gives the lowest uncertainty whilst also including the Crab pulsar. These are given for observation times, $\mathrm{T}_{\mathrm{obs}}$, of $5 \times 10^{3}$ and $5 \times 10^{4} \mathrm{~s}$. For the latter pulsar-set with $\mathrm{T}_{\text {obs }}=5 \times 10^{4} \mathrm{~s}$, the position uncertainty budget is lower by a factor of $\sim 2$ at ecliptic coordinates given by $x=0 \mathrm{AU}, y=30 \mathrm{AU}$ and $z=0 \mathrm{AU}$. It is found that although the former pulsar set gives the lowest position uncertainty of any three pulsar set for $\mathrm{T}_{\mathrm{obs}}=$ $5 \times 10^{4} \mathrm{~s}$, PSRs B1937+21, B1821-24 and J1012 +5307 give the lowest uncertainty for $\mathrm{T}_{\text {obs }}=5 \times 10^{3} \mathrm{~s}$ corresponding to $80 \mathrm{~km}$. The contribution due to pulsar position uncertainties in this case is $25 \mathrm{~km}$. A consequence of using the simulated range errors is that the optimum pulsar set depends on the observation time. Table 4 presents the relevant uncertainty budgets when using four pulsars and Table 5 for a single pulsar in the cases of PSR B1937+21 and the Crab pulsar.

The simulations are applicable to scenarios related to interplanetary navigation, which accounts for the majority of deep space missions. The approaches described can be used to evaluate uncertainties for any velocity vector, including one inclined to 
Table 3 Spacecraft position and velocity uncertainty budgets for absolute navigation at ecliptic coordinates $x=30 \mathrm{AU}, y=0 \mathrm{AU}$ and $z=0 \mathrm{AU}$ using the three-pulsar set giving the lowest position uncertainties $\left(\right.$ for $\mathrm{T}_{\mathrm{obs}}=$ $5 \times 10^{4} \mathrm{~s}$ ) and for the three pulsar-set giving the lowest uncertainties whilst also including the Crab pulsar, PSR $\mathrm{B} 0531+21$

\begin{tabular}{|c|c|c|c|c|c|c|c|c|}
\hline \multirow[t]{3}{*}{ Uncertainty Source } & \multicolumn{4}{|c|}{ PSR B1937+21, B1821-24, J0437-4715 } & \multicolumn{4}{|c|}{ PSR B1937+21, B0531+21, J0437-4715 } \\
\hline & \multicolumn{2}{|c|}{$\begin{array}{l}\text { Position Uncertainty } \\
\text { Contribution }(\mathrm{km})\end{array}$} & \multicolumn{2}{|c|}{$\begin{array}{l}\text { Velocity Uncertainty } \\
\text { Contribution }\left(\mathrm{ms}^{-1}\right)\end{array}$} & \multicolumn{2}{|c|}{$\begin{array}{l}\text { Position Uncertainty } \\
\text { Contribution }(\mathrm{km})\end{array}$} & \multicolumn{2}{|c|}{$\begin{array}{l}\text { Velocity Uncertainty } \\
\text { Contribution }\left(\mathrm{ms}^{-1}\right)\end{array}$} \\
\hline & $\begin{array}{l}\mathrm{T}_{\mathrm{obs}}= \\
5 \times 10^{4} \mathrm{~s}\end{array}$ & $\begin{array}{l}\mathrm{T}_{\mathrm{obs}}= \\
5 \times 10^{3} \mathrm{~s}\end{array}$ & $\begin{array}{l}\mathrm{T}_{\mathrm{obs}}= \\
5 \times 10^{4} \mathrm{~s}\end{array}$ & $\begin{array}{l}\mathrm{T}_{\mathrm{obs}}= \\
5 \times 10^{3} \mathrm{~s}\end{array}$ & $\begin{array}{l}\mathrm{T}_{\mathrm{obs}}= \\
5 \times 10^{4} \mathrm{~s}\end{array}$ & $\begin{array}{l}\mathrm{T}_{\mathrm{obs}}= \\
5 \times 10^{3} \mathrm{~s}\end{array}$ & $\begin{array}{l}\mathrm{T}_{\mathrm{obs}}= \\
5 \times 10^{4} \mathrm{~s}\end{array}$ & $\begin{array}{l}\mathrm{T}_{\mathrm{obs}}= \\
5 \times 10^{3} \mathrm{~s}\end{array}$ \\
\hline $\begin{array}{l}\text { Instrument and } \\
\text { timing model }\end{array}$ & 30 & 120 & 0.6 & 25 & 30 & 110 & 0.6 & 20 \\
\hline Pulsar position & 20 & 20 & $0.1 \times 10^{-3}$ & $0.1 \times 10^{-3}$ & 90 & 90 & $0.6 \times 10^{-3}$ & $0.6 \times 10^{-3}$ \\
\hline Total uncertainty & 35 & 120 & 0.6 & 25 & 100 & 140 & 0.6 & 20 \\
\hline
\end{tabular}

the plane of the ecliptic, and any spacecraft location within the Solar System and to some extent beyond.

\subsection{Summary of navigation and timing uncertainties}

The PVT uncertainties derived in this section are summarised in Table 6. The spacecraft positioning uncertainty component due to the pulsar position uncertainty can be calculated for other ranges using the uncertainty budgets given. To do this the ratio of the required range to $30 \mathrm{AU}$ is multiplied by the given value of this component at $30 \mathrm{AU}$. In this way, results for a spacecraft at a distance of 1 AU from the SSB are also shown in Table 6 for comparison.

It should be noted that the performance results given at 1 AU in Table 6 are for the best-performing pulsar-sets determined at ecliptic coordinates given by $x=30 \mathrm{AU}$, $y=0 \mathrm{AU}$ and $z=0 \mathrm{AU}$. Other pulsar sets may have similar or marginally lower

Table 4 Spacecraft position, clock time-offset, velocity uncertainty and time drift rate uncertainty budgets for absolute navigation at ecliptic coordinates $x=30 \mathrm{AU}, y=0 \mathrm{AU}$ and $z=0 \mathrm{AU}$ using the four pulsar-set giving the lowest position uncertainties

\begin{tabular}{|c|c|c|c|c|c|c|c|c|}
\hline \multirow[t]{3}{*}{ Uncertainty Source } & \multicolumn{8}{|c|}{ PSR B1937+21, B1821-24, J1012+5307 and J0437-4715 } \\
\hline & \multicolumn{2}{|c|}{$\begin{array}{l}\text { Position } \\
\text { Uncertainty } \\
\text { Contribution }(\mathrm{km})\end{array}$} & \multicolumn{2}{|c|}{$\begin{array}{l}\text { Time-Offset } \\
\text { Uncertainty } \\
\text { Contribution (s) }\end{array}$} & \multicolumn{2}{|c|}{$\begin{array}{l}\text { Velocity } \\
\text { Uncertainty } \\
\text { Contribution }\left(\mathrm{ms}^{-1}\right)\end{array}$} & \multicolumn{2}{|c|}{$\begin{array}{l}\text { Time Drift } \\
\text { Rate Uncertainty } \\
\text { Contribution }\left(\mathrm{ss}^{-1}\right)\end{array}$} \\
\hline & $\begin{array}{l}\mathrm{T}_{\mathrm{obs}}= \\
5 \times 10^{4} \mathrm{~s}\end{array}$ & $\begin{array}{l}\mathrm{T}_{\mathrm{obs}}= \\
5 \times 10^{3} \mathrm{~s}\end{array}$ & $\begin{array}{l}\mathrm{T}_{\mathrm{obs}}= \\
5 \times 10^{4} \mathrm{~s}\end{array}$ & $\begin{array}{l}\mathrm{T}_{\mathrm{obs}}= \\
5 \times 10^{3} \mathrm{~s}\end{array}$ & $\begin{array}{l}\mathrm{T}_{\mathrm{obs}}= \\
5 \times 10^{4} \mathrm{~s}\end{array}$ & $\begin{array}{l}\mathrm{T}_{\mathrm{obs}}= \\
5 \times 10^{3} \mathrm{~s}\end{array}$ & $\begin{array}{l}\mathrm{T}_{\mathrm{obs}}= \\
5 \times 10^{4} \mathrm{~s}\end{array}$ & $\begin{array}{l}\mathrm{T}_{\mathrm{obs}}= \\
5 \times 10^{3} \mathrm{~s}\end{array}$ \\
\hline $\begin{array}{l}\text { Instrument and } \\
\text { timing model }\end{array}$ & 30 & 80 & $40 \times 10^{-6}$ & $100 \times 10^{-6}$ & 0.6 & 15 & $1 \times 10^{-9}$ & $20 \times 10^{-9}$ \\
\hline Pulsar position & 20 & 20 & $10 \times 10^{-6}$ & $10 \times 10^{-6}$ & $0.1 \times 10^{-3}$ & $0.1 \times 10^{-3}$ & $6 \times 10^{-14}$ & $6 \times 10^{-14}$ \\
\hline Total uncertainty & 35 & 80 & $40 \times 10^{-6}$ & $100 \times 10^{-6}$ & 0.6 & 15 & $1 \times 10^{-9}$ & $20 \times 10^{-9}$ \\
\hline
\end{tabular}


Table 5 Spacecraft position and velocity uncertainty budgets for the delta-correction method at ecliptic coordinates $x=30 \mathrm{AU}, y=0 \mathrm{AU}$ and $z=0 \mathrm{AU}$ using a single pulsar with PSR B1937+21 and B0531+21

\begin{tabular}{|c|c|c|c|c|c|c|c|c|}
\hline \multirow[t]{3}{*}{ Uncertainty Source } & \multicolumn{4}{|c|}{ PSR B1937+21 } & \multicolumn{4}{|c|}{ PSR B0531+21 } \\
\hline & \multicolumn{2}{|c|}{$\begin{array}{l}\text { Position } \\
\text { Uncertainty } \\
\text { Contribution }(\mathrm{km})\end{array}$} & \multicolumn{2}{|c|}{$\begin{array}{l}\text { Velocity } \\
\text { Uncertainty } \\
\text { Contribution }\left(\mathrm{ms}^{-1}\right)\end{array}$} & \multicolumn{2}{|c|}{$\begin{array}{l}\text { Position } \\
\text { Uncertainty } \\
\text { Contribution (km) }\end{array}$} & \multicolumn{2}{|c|}{$\begin{array}{l}\text { Velocity } \\
\text { Uncertainty } \\
\text { Contribution }\left(\mathrm{ms}^{-1}\right)\end{array}$} \\
\hline & $\begin{array}{l}\mathrm{T}_{\mathrm{obs}}= \\
5 \times 10^{4} \mathrm{~s}\end{array}$ & $\begin{array}{l}\mathrm{T}_{\mathrm{obs}}= \\
5 \times 10^{3} \mathrm{~s}\end{array}$ & $\begin{array}{l}\mathrm{T}_{\mathrm{obs}}= \\
5 \times 10^{4} \mathrm{~s}\end{array}$ & $\begin{array}{l}\mathrm{T}_{\mathrm{obs}}= \\
5 \times 10^{3} \mathrm{~s}\end{array}$ & $\begin{array}{l}\mathrm{T}_{\mathrm{obs}}= \\
5 \times 10^{4} \mathrm{~s}\end{array}$ & $\begin{array}{l}\mathrm{T}_{\mathrm{obs}}= \\
5 \times 10^{3} \mathrm{~s}\end{array}$ & $\begin{array}{l}\mathrm{T}_{\mathrm{obs}}= \\
5 \times 10^{4} \mathrm{~s}\end{array}$ & $\begin{array}{l}\mathrm{T}_{\mathrm{obs}}= \\
5 \times 10^{3} \mathrm{~s}\end{array}$ \\
\hline $\begin{array}{l}\text { Instrument and } \\
\text { timing model }\end{array}$ & 1.4 & 4.6 & 0.03 & 1 & 3.7 & 5.5 & 0.07 & 1 \\
\hline Pulsar position & 0.7 & 0.7 & $6 \times 10^{-6}$ & $6 \times 10^{-6}$ & 70 & 70 & $500 \times 10^{-6}$ & $500 \times 10^{-6}$ \\
\hline Total uncertainty & 1.5 & 4.7 & 0.03 & 1 & 70 & 70 & 0.07 & 1 \\
\hline
\end{tabular}

uncertainties at $1 \mathrm{AU}$ from the SSB. Furthermore, the analysis here uses pulsar position uncertainties currently available in the literature. These will improve in future by different amounts depending on the pulsar, as new measurements become available.

\section{Available and future X-Ray technology}

XNAV requires high signal-to-noise timing observations of one or more pulsars to derive a spacecraft position estimate. There is a trade-off between the ability to generate high signal-to-noise observations of this type and the requirement of a positioning system to be deployable as a spacecraft subsystem, where mass, volume and power consumption are tightly constrained. XNAV requires a time resolution of $<1 \mu \mathrm{s}$ (a significant performance improvement is possible if $100 \mathrm{~ns}$ is achievable) and a high collecting area, the value of which depends upon whether the instrument is imaging or not. Ideally the system would be able to simultaneously observe up to three or four pulsars which are widely separated on the sky. In this section, we first describe the required characteristics of instrumentation for an XNAV system. We then discuss some of the design and development challenges that need to be overcome. Finally, we discuss the technology trade-offs and optimisations that need to be considered regarding instrument performance.

\subsection{Observing pulsars for X-ray astronomy}

X-ray instruments used in astrophysics, in general, use single photon-counting detectors; hence are well suited for use in timing studies [28]. Literature on instrumentation for high time-resolution pulsar astrophysics is dominated by collimated instruments (e.g. [42]). However, in recent studies of the XNAV concept [8] imaging instrumentation is proposed to allow the use of more capable solid-state focal plane devices which offer higher positioning accuracy due to reduced background and hence higher signal-to-noise. 
Table 6 A summary of the PVT uncertainties for a spacecraft located in the ecliptic plane at a distance of 30 $\mathrm{AU}$ and $1 \mathrm{AU}$ from the SSB in the direction of zero degrees ecliptic longitude and latitude

\begin{tabular}{|c|c|c|c|c|c|}
\hline \multirow[t]{2}{*}{ Navigation strategy \& pulsars } & \multirow[t]{2}{*}{ Parameter } & \multicolumn{2}{|l|}{$30 \mathrm{AU}$} & \multicolumn{2}{|l|}{$1 \mathrm{AU}$} \\
\hline & & $\begin{array}{l}\mathrm{T}_{\mathrm{obs}}= \\
5 \times 10^{4} \mathrm{~s}\end{array}$ & $\begin{array}{l}\mathrm{T}_{\mathrm{obs}}= \\
5 \times 10^{3} \mathrm{~s}\end{array}$ & $\begin{array}{l}\mathrm{T}_{\mathrm{obs}}= \\
5 \times 10^{4} \mathrm{~s}\end{array}$ & $\begin{array}{l}\mathrm{T}_{\mathrm{obs}}= \\
5 \times 10^{3} \mathrm{~s}\end{array}$ \\
\hline \multirow{2}{*}{$\begin{array}{l}\text { (i) Absolute navigation using } \\
\text { three pulsars } \\
\text { PSR B1937+21, B1821-24, } \\
\text { J0437-4715 }\end{array}$} & Position & $35 \mathrm{~km}$ & $120 \mathrm{~km}$ & $30 \mathrm{~km}$ & $120 \mathrm{~km}$ \\
\hline & Velocity & $0.6 \mathrm{~ms}^{-1}$ & $25 \mathrm{~ms}^{-1}$ & $0.6 \mathrm{~ms}^{-1}$ & $25 \mathrm{~ms}^{-1}$ \\
\hline \multirow{2}{*}{$\begin{array}{l}\text { (ii) Absolute navigation using } \\
\text { three pulsars } \\
\text { PSR B1937+21, B1821-24, } \\
\text { J1012+5307 }\end{array}$} & Position & $50 \mathrm{~km}$ & $80 \mathrm{~km}$ & $45 \mathrm{~km}$ & $80 \mathrm{~km}$ \\
\hline & Velocity & $0.9 \mathrm{~ms}^{-1}$ & $15 \mathrm{~ms}^{-1}$ & $0.9 \mathrm{~ms}^{-1}$ & $15 \mathrm{~ms}^{-1}$ \\
\hline \multirow{2}{*}{$\begin{array}{l}\text { (iii) Absolute navigation using } \\
\text { three pulsars } \\
\text { PSR B1937+21, B0531+21, } \\
\text { J0437-4715 }\end{array}$} & Position & $100 \mathrm{~km}$ & $140 \mathrm{~km}$ & $30 \mathrm{~km}$ & $110 \mathrm{~km}$ \\
\hline & Velocity & $0.6 \mathrm{~ms}^{-1}$ & $20 \mathrm{~ms}^{-1}$ & $0.6 \mathrm{~ms}^{-1}$ & $20 \mathrm{~ms}^{-1}$ \\
\hline \multirow{4}{*}{$\begin{array}{l}\text { (iv) Absolute navigation using } \\
\text { four pulsars } \\
\text { PSR B1937+21, B1821-24, } \\
\text { J1012+5307, J0437-4715 }\end{array}$} & Position & $35 \mathrm{~km}$ & $80 \mathrm{~km}$ & $30 \mathrm{~km}$ & $80 \mathrm{~km}$ \\
\hline & Clock time-offset & $40 \times 10^{-6} \mathrm{~s}$ & $\begin{array}{l}100 \times 10^{-6} \\
\mathrm{~s}\end{array}$ & $40 \times 10^{-6} \mathrm{~s}$ & $\begin{array}{l}100 \times 10^{-6} \\
\mathrm{~s}\end{array}$ \\
\hline & Velocity & $0.6 \mathrm{~ms}^{-1}$ & $15 \mathrm{~ms}^{-1}$ & $0.6 \mathrm{~ms}^{-1}$ & $15 \mathrm{~ms}^{-1}$ \\
\hline & Clock time-drift rate & $\begin{array}{c}1 \times 10^{-9} \\
\mathrm{ss}^{-1}\end{array}$ & $\begin{array}{c}20 \times 10^{-9} \\
\mathrm{ss}^{-1}\end{array}$ & $\begin{array}{c}1 \times 10^{-9} \\
\mathrm{ss}^{-1}\end{array}$ & $\begin{array}{c}20 \times 10^{-9} \\
\mathrm{ss}^{-1}\end{array}$ \\
\hline \multirow{2}{*}{$\begin{array}{l}\text { (v) Delta-correction using } \\
\text { a single pulsar with } \\
\text { PSR B1937+21 }\end{array}$} & $\begin{array}{l}\text { Position (in direction } \\
\text { of pulsar) }\end{array}$ & $1.5 \mathrm{~km}$ & $4.7 \mathrm{~km}$ & $1.4 \mathrm{~km}$ & $4.6 \mathrm{~km}$ \\
\hline & $\begin{array}{l}\text { Velocity (in direction } \\
\text { of pulsar) }\end{array}$ & $0.03 \mathrm{~ms}^{-1}$ & $1 \mathrm{~ms}^{-1}$ & $0.03 \mathrm{~ms}^{-1}$ & $1 \mathrm{~ms}^{-1}$ \\
\hline \multirow{2}{*}{$\begin{array}{l}\text { (vi) Delta-correction using } \\
\text { a single pulsar with } \\
\text { PSR B0531+21 }\end{array}$} & $\begin{array}{l}\text { Position (in direction } \\
\text { of pulsar) }\end{array}$ & $70 \mathrm{~km}$ & $70 \mathrm{~km}$ & $4.5 \mathrm{~km}$ & $6 \mathrm{~km}$ \\
\hline & $\begin{array}{l}\text { Velocity (in direction } \\
\text { of pulsar) }\end{array}$ & $0.07 \mathrm{~ms}^{-1}$ & $1 \mathrm{~ms}^{-1}$ & $0.07 \mathrm{~ms}^{-1}$ & $1 \mathrm{~ms}^{-1}$ \\
\hline
\end{tabular}

These may be achievable with a focussing instrument with an effective area of $0.005 \mathrm{~m}^{2}$ and are representative of 1-sigma

Orbiting observatories dedicated to high time resolution astronomy are designed to fulfil multiple scientific goals and as such are not optimised specifically for the XNAV principle. They are generally dedicated payloads (or at least a dominant instrument) on a low earth orbit mission. For a practical XNAV system to be developed, instrumentation must be designed to meet the following requirements:

i. large collecting area to minimise statistical noise in observations of faint sources;

ii. high timing accuracy - typically $<1 \mu$ s time stamping uncertainty;

iii. low background noise from internal and external sources (such as the detector intrinsic background, particle induced signals, cosmic rays and the diffuse X-ray background);

iv. Engineering constraints (low mass, volume, power consumption). 
But crucially, the system must also be implementable as a realistic spacecraft subsystem, hence a particular emphasis on requirement iv in what follows.

\subsection{The advantage of imaging}

The primary scientific justification for true X-ray imaging, rather than scanning with a collimated instrument over a specific field of view is to reduce background. In a true imaging system, the signal collecting area is greater than the collecting area relevant for background noise. In such a system, the relevant area for (non-sky ${ }^{5}$ ) background collection is equal to the size of the optic PSF, rather than the whole area of the detector (as would be the case for a collimated instrument). The effective collecting area for the signal is governed principally by the aperture of the optic and its focal length. ${ }^{6}$ Combining these effects, the signal-to-noise ratio of an imaging system is improved by the "imaging advantage" (I) defined as:

$$
I=\sqrt{\frac{A_{e f f}}{A_{B G}}},
$$

where $A_{\text {eff }}$ is the effective collecting area of the optic and $A_{B G}$, the detection cell size in the detector plane (governed by the optic PSF). This assumes that the sky background (diffuse X-ray background and confusion limited point sources) is negligible, which may not be true depending on the characteristics of the pulsar. In such a case, the background contribution from the sky must be accounted for when assessing the improvement in signal-to-noise as a result of imaging.

Of course, there are additional benefits of imaging which range from engineering parameters (smaller detectors: less particle shielding, simpler thermal control, simplified focal plane design) to scientific observation advantages such as a reduction in "sky background" from diffuse X-ray emission and unresolvable point sources on the sky. The only significant disadvantage of an imaging system is the need to design a sufficiently accurate pointing mechanism capable of steering an $\sim 1 \mathrm{~m}$ focal length telescope to acquire the pulsar of interest.

A collimated XNAV system would require a minimum focal plane area $\sim 10^{3} \mathrm{~cm}^{2}$. Assuming a large-area collimated silicon detector were to be used, the cooling of the detectors and shielding them from high energy particles would be difficult to achieve as a small, optimised, XNAV subsystem. Also, the overall system complexity would be considerable, particularly when the requirement to perform photon time-tagging with microsecond uncertainty over the whole detector area is considered. While a much larger system has been studied for the Large Observatory for X-ray Timing (LOFT) mission [27] as the dominant instrument of a large satellite [82], it is difficult to foresee a time when this level of complexity could be integrated into a spacecraft navigation subsystem, even though the LOFT hardware is well-suited to the XNAV principle.

\footnotetext{
${ }^{5}$ Note that the sky background is proportional to the optic effective area as well as the relevant focal plane area.

${ }^{6}$ The maximum useful aperture is driven by the critical angle for X-ray reflection, hence the dependence on focal length.
} 
Recent developments in low-mass optics (e.g. [29, 81]) have enabled the design of compact imagers, requiring greatly reduced spacecraft resources compared to established X-ray optics technologies. This technological advancement offers the possibility of implementing a true imaging X-ray telescope in a resource envelope commensurate with a sub-system on a deep space vehicle. The first example of such an instrument to be deployed in deep space will be the Mercury Imaging Xray Spectrometer (MIXS) [29], one of 11 instruments on the ESA/JAXA BepiColombo mission to Mercury [9]. The telescope, MIXS-T could be considered a "pathfinder" for XNAV technology as it is designed to very similar engineering constraints. Figure 6 shows the key components of the MIXS-T instrument.

\subsection{Design and development challenges for an XNAV instrument}

Below we discuss the principal design and development challenges that must be addressed in order to realise an operational XNAV instrument.

\subsubsection{Observing strategy}

As described in Section 2.2.2, the most capable XNAV system would incorporate simultaneous observations of a minimum of three or four pulsars. However, the complexity of a multiple field-of-view X-ray instrument, with separate telescopes that are independently steerable would be high, making it challenging to implement. It is much more reasonable to envisage a single pointed instrument being deployed on a deep space mission. Similarly, ideal XNAV observations would be long, uninterrupted stares towards targets of interest. Although there are no intrinsic limits on the observation duration set by the detector system, practical limitations will come from specific mission scenarios. For instance, the instrument will have pointing constraints relating to solar-system objects (typically; the Sun, nearby planets and moons) that will limit the available sky and the observation duration. X-ray instruments have a solar avoidance angle typically $\sim 30-40^{\circ}$, so the fraction of the sky that is not visible at any given time may be significant.

\subsubsection{Optics}

MicroChannel-Plate (MCP) optics are the key enabling technology for low-mass $(<10 \mathrm{~kg})$ imaging X-ray instruments (e.g. [29, 54]). MIXS uses a radially packed array of microscopic square pores to simulate the mirror shells of a more traditional Wolter-I
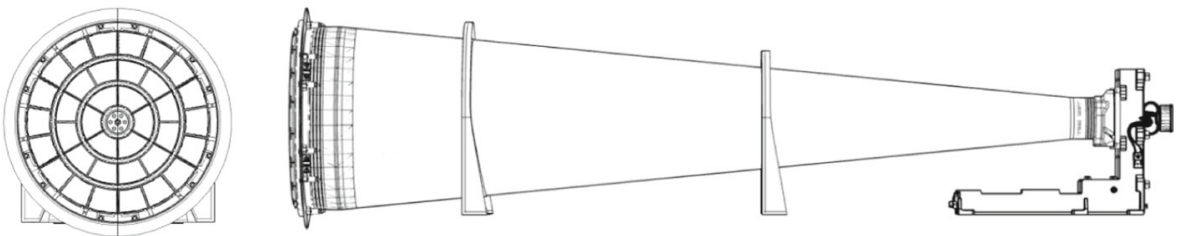

Fig. 6 Diagram showing the key components of the BepiColombo MIXS-T imaging Wolter-I telescope comprising a microchannel plate optic (left), a telescope tube assembly and a focal plane assembly containing a DEPFET active pixel sensor detector (right) 
telescope and meets similar resource constraints as an XNAV system. The optics themselves are made of lead-glass septal walls, the result of a manufacturing process which etches millions of microscopic pores from the glass. For MCP optics, these channels are square in cross-section and can be coated with high atomic number material to increase the reflectivity of the channel walls, improving the collecting power of the telescope. The total mass of the MIXS instrument is only $\sim 10 \mathrm{~kg}$ including the electronics boxes, mechanics and a collimated instrument, MIXS-C [29], which is of no direct relevance to XNAV. The telescope has a focal length of $1 \mathrm{~m}$ and offers an effective area of $\sim 0.005 \mathrm{~m}^{2}$ with a high performance silicon DEPFET active pixel sensor detector. The detector is optimised for spectroscopy and provides almost Fanolimited energy resolution [51]. Hence, it does not operate at the time resolution required for XNAV, but could be replaced by a suitable detector, as discussed below.

Significant simplification and optimisation of the MIXS design is possible for XNAV. Either a narrow field lobster-eye telescope [1] or a cylindrical microchannel plate approximation to the Kirkpatrick-Baez (KB) geometry [45] offers a simpler optical design with higher spatial resolution (and hence lower background) than the current $\sim 7-10$ arcminutes. Proof-of-principal experiments with a partial prototype of the KB system have demonstrated $\sim 3$ arcminutes FWHM. A programme of work to demonstrate the limiting performance of a lobster-eye optic is underway at the University of Leicester and Photonis France, SAS. It is realistic to expect an optic with large collecting area and improved imaging resolution to emerge from a dedicated research and development programme.

Note that the simulations of XNAV performance reported here are based on MIXSlike hardware, but assume an improved PSF of 3.5 arcminutes FWHM. It should be noted that while existing hardware can already meet the major goals of XNAV, improvements in the optic presented here lead directly to smaller range errors (due to better rejection of background by a higher quality optic). The performance improvement varies for different pulsars based on their brightness. A better optic is insignificant for the Crab, but offers improvements up to $\sim 50 \%$ for the weaker sources such as PSR $\mathrm{J} 1012+5307$.

\subsubsection{Detectors}

Considering available detector technologies, the optimum existing device for XNAV is an extremely fast, single pixel, Silicon Drift Detector (SDD), examples of which are available from AMPTEK Inc. and PN Detector GmbH. For example, AMPTEK's super-fast SDD offers excellent energy resolution, close to the fundamental Fano limit, if operated with long peaking times, or, if operated with very fast peaking times $(\sim 0.2 \mu \mathrm{s})$, the energy resolution is degraded, but the time resolution is improved. As energy resolution is not a fundamental driver for XNAV - it only allows rejection of background based on photon energy - this degraded energy resolution is not a significant driver and fast shaping time is preferred.

The absolute timing accuracy of the device is not only a function of the peaking time. As the X-ray can strike anywhere in the active detector volume, and the charge generated by the X-ray interaction is drifted to a readout node, the time-delay variation caused by the drifting of charge will increase the error in measuring the absolute arrival time of the photon. To match the expected performance of the optic and detector a 
diode of diameter $2.5 \mathrm{~mm}$ is required and the quantum efficiency should be maximised in the range $\sim 0.5-8 \mathrm{keV}$. For such a large pixel, it is unlikely to be able to achieve a time resolution of better than $\sim 0.5 \mu \mathrm{s}$ due to the physics of drifting charge to the readout. By using a pixelated device with small pixels, the drift time is reduced and higher limiting time resolution is possible; such devices are under development for applications in high flux X-ray sources such as free electron lasers with time resolution in the low hundreds of ns (e.g. [60]), a development that could be important for future XNAV studies.

\subsubsection{On-board clock and timing}

The requirements for maintaining on-board timing precision are stringent, but not outside the scope of existing technology. For instance, the clocks on-board the Galileo satellites [23] would offer precision considerably greater than needed for an XNAV system during single observations of up to a few days. The complication in provision of a timing reference is in maintaining high accuracy absolute time relative to terrestrial time scales such as TAI (Temps Atomique International) over the lifetime of a deep space mission. This would be required when positioning in the direction of a single pulsar or in order to reduce the numbers of pulsars to be observed from four to three for three-dimensional positioning. Pulse TOA measurements need to be obtained relative to terrestrial time scales, ideally to better than $300 \mathrm{~ns}$, without timing drift becoming a significant error source. In the next decade, it is possible that a clock with sufficient stability may be demonstrated in space. For example, NASA is currently developing a deep space mercury ion-trap atomic clock [72]. Without such a clock, it would be necessary to employ a time transfer link to Earth using the DSN to periodically calibrate the on-board clock relative to, for example, TAI. If in the future an instrument with a significantly improved TOA measurement error was to be employed, a clock with commensurately lower uncertainty would be required. For example, a space optical clock similar to those currently under development (e.g. [67]), could be of benefit to a long-duration advanced space experiment.

\subsection{Technology trade-offs and optimisation}

Detailed system design is outside the scope of this paper. However, it is possible to parameterise the performance of the instrument in terms of its focal length (hence mass) and key XNAV figures of merit such as the signal-to-noise ratio and the range error contribution due to the instrument. The focal length influences these performance parameters via its relationship to focal-plane scale and effective area [1].

In Fig. 7 (right panel) this approach is used to show that the range error decreases with increasing instrument focal length (and hence collecting area), showing that instruments with a focal length of $25 \mathrm{~cm}$ may be useful for the Crab pulsar and PSR $\mathrm{B} 1937+21$, but for a navigation system to be generally applicable to fainter pulsars, a longer focal length (e.g. the baseline $1 \mathrm{~m}$ described below) is required. Figure 7 (left) shows that, except for the Crab pulsar, the signal to noise ratio per unit mass increases for longer focal lengths, implying that a larger (more capable) telescope provides a more mass efficient solution with longer focal lengths. Hence, a larger telescope is preferable to an approach reliant on multiple, co-pointing telescopes to increase the 


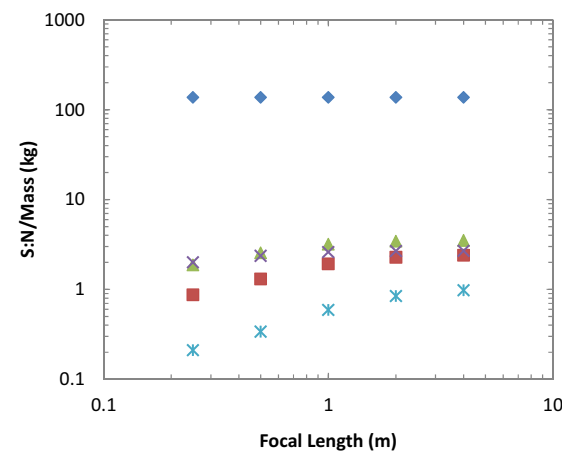

-B0531+21 B1821-24 A B1937+21 × 0437-4715 *J1012+5307

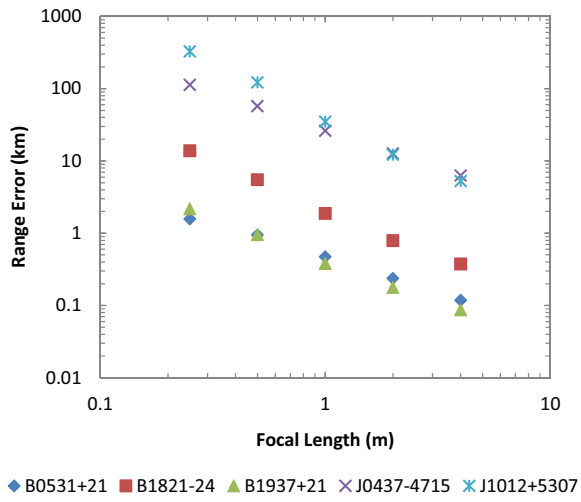

Fig. 7 Left- the Signal-to-Noise ratio (S:N) per unit mass versus focal length for 5 pulsars assuming an observation of $5 \times 10^{4}$ s. Right- the range error obtained using the analytic formula (see Section 3.3.1) as a function of focal length for 5 pulsars. The different order of the pulsars in the two panels is a result of the relative importance of signal strength, the pulse width and pulse period of the various pulsars

collecting area (assuming the dominant consideration for spacecraft resource planning is mass). The optimum, practically realisable system for XNAV is likely to be based on the light weight optics described here, specifically, a single (steerable) KB optic coupled to an SDD detector.

The properties and predicted performance of such a telescope are described in Table 7. In assessing the spacecraft resources required for this proposed device a number of assumptions are made. It is assumed that a (currently) low maturity instrument based on a KB design can be achieved for a similar mass as the MIXS-T Wolter I design. To cover the risk of this low maturity, a small margin $(0.7 \mathrm{~kg})$ is added to the predicted optic mass. The $6.4 \mathrm{~kg}$ detector and housing includes a conservative estimate of $5.5 \mathrm{~kg}$ for a radiation shield to protect the detectors from solar protons and other ionizing radiation. The DPU mass is estimated based on SSTL's OBC750 LEO unit.

Table 7 also provides an estimate of the power consumption for the device which includes; the DPU $(10 \mathrm{~W})$, detector and front-end electronics $(0.25 \mathrm{~W})$, analogue electronics $(2 \mathrm{~W})$, PSU efficiency $(70 \%)$. The power estimate is based on the experience of MIXS and excludes thermal control (assumed to be provided by a spacecraft radiator).

\section{Discussion}

As described in Curkendall and Border 2013, a single one of the antennas used in NASA's DSN can be used to carry out two-way ranging to a spacecraft to measure its distance along the line-of-sight to the Earth extremely precisely to an uncertainty of a few metres. Furthermore, pairs of antennas can also be used at a time for very long baseline interferometry measurements referred to as Delta-Differential One-Way Ranging (Delta-DOR). By doing this, the DSN can currently be used to obtain tracking uncertainties approaching $1 \mathrm{nrad}$, equivalent to $150 \mathrm{~m}$ and $4.5 \mathrm{~km}$ in the plane of the sky for spacecraft distances of $1 \mathrm{AU}$ and $30 \mathrm{AU}$ from Earth respectively. This means that uncertainties of $150 \mathrm{~m}$ are possible at an Earth-Mars distance of 1 AU. At a range 
Table 7 Summary of the characteristics of an imaging XNAV system (excluding thermal control and telescope pointing mechanisms) based on an instrument similar to MIXS-T and a more optimised KB optic

\begin{tabular}{llll}
\hline Parameter & MIXS-T & KB Optic & Units \\
\hline $\begin{array}{l}\text { Optic Performance } \\
\text { Optic effective area }\end{array}$ & 50 & & \\
$\begin{array}{l}\text { Focal plane scale } \\
\text { Mass estimate }\end{array}$ & 3.44 & 80 & $\mathrm{~cm}^{2} @ 1 \mathrm{keV}$ \\
Optic mass & & 3.44 & $\mathrm{arcmin} / \mathrm{mm}$ \\
Detector/housing & 1.8 & & $\mathrm{~kg}$ \\
DPU & 6.4 & 2.5 & $\mathrm{~kg}$ \\
PSU & 1.5 & 6.4 & $\mathrm{~kg}$ \\
Harness and misc. & 1 & 1.5 & $\mathrm{~kg}$ \\
TOTAL & 1 & 1 & $\mathrm{~kg}$ \\
Volume estimate & 11.7 & 1 & $\mathrm{~kg}$ \\
Telescope: & & 12.4 & $\mathrm{~mm}$ \\
DPU: & $250 \times 250 \times 1000$ & $260 \times 260 \times 1000$ & \\
PSU: & $320 \times 170 \times 55$ & $320 \times 170 \times 55$ & \\
Detector Parameters & $320 \times 170 \times 55$ & $320 \times 170 \times 55$ & \\
Type & & & \\
Time resolution & APD/SDD & APD/SDD & \\
Cooling required & $1(0.3$ goal & $1(0.3$ goal $)$ & $\mu \mathrm{W}$ \\
Detector active area & -20 & -20 & ${ }^{3} \mathrm{C}$ \\
Quantum efficiency & 0.049 & 0.049 & $\mathrm{~cm}^{2}$ \\
Power consumption estimate & $>90$ & $>90$ & $\%$ \\
\hline & 15.9 & 15.9 & \\
\hline
\end{tabular}

of $30 \mathrm{AU}$ and for $\mathrm{T}_{\mathrm{obs}}=5 \times 10^{4} \mathrm{~s}$, three-dimensional XNAV positioning uncertainties for an improved PSF version of the BepiColombo-MIXS instrument given in Table 6 are generally $\sim$ one order of magnitude greater than those described above for the DSN, although with the potential for being somewhat lower in the direction of PSR B1937+ 21. However, with the exception of the cases when the Crab pulsar is used, these uncertainties could be achieved without the need to communicate with Earth to update the pulsar's timing model for up to 3 months. In the case of Delta-DOR for the ESTRACK network, the tracking uncertainties are currently 6 to $15 \mathrm{nrad}$ [41], although there are ongoing studies with the aim of improving these to $1 \mathrm{nrad}$. The XNAV positioning uncertainties are generally of the same order at a range of $30 \mathrm{AU}$, although more than an order of magnitude lower in the direction of PSR B1937+21. A key advantage for XNAV is that, unlike the use of the ESTRACK or DSN systems for navigation, it could allow a greater level of spacecraft autonomy because it requires significantly less communication with Earth-based systems.

With $\mathrm{T}_{\mathrm{obs}}=5 \times 10^{4} \mathrm{~s}$, the effects of the first component due to instrument and timing model uncertainties and the second component due to pulsar position uncertainties are of a similar order of tens of kilometres for each of the three and four pulsar cases at a distance of $30 \mathrm{AU}$ from the SSB. For the single and three-pulsar cases involving the 
Crab pulsar, the effect of pulsar position uncertainty can be the larger component by far for some spacecraft locations. For $\mathrm{T}_{\text {obs }}>1 \times 10^{5} \mathrm{~s}(\sim 1$ day $)$, the effect of pulsar position uncertainties would start to dominate in the other cases. Consequently, this component may be the limiting factor in determining spacecraft position. However, if the latency period of the timing model for each pulsar, apart from for the Crab pulsar, was to become on the order of years then the effect of timing model uncertainties would begin to dominate. In the case of the Crab pulsar, this would apply if the latency period was on the order of months. If pulsar position uncertainties reduce in future, the spacecraft position uncertainties would also reduce. However, in the case of the velocity uncertainties, these are always dominated by the instrument and timing model uncertainties for the above $\mathrm{T}_{\text {obs }}$ values.

From Eqs B.2, B.5 and B.8 (see Appendix B), it can be seen that for the XNAV positioning error, the component due to pulsar position error increases with spacecraft range from the SSB. From Table 6, it can be seen that the spacecraft position uncertainties are generally of the same order at ranges of 1 and $30 \mathrm{AU}$ for a given $\mathrm{T}_{\text {obs }}$ value of $5 \times 10^{4}$ or $5 \times 10^{3} \mathrm{~s}$. In the single pulsar case of the $\mathrm{Crab}$, the position uncertainties at $1 \mathrm{AU}$ are much lower due to the relatively high pulsar position uncertainty of this pulsar. Apart from for this particular case, the spacecraft position uncertainties at 1 AU are heavily dominated by the instrument and timing model uncertainties.

Out of the pulsars considered, the three-pulsar set PSR B1937+21, B1821-24 and J0437-4715 is found to show the lowest spacecraft position uncertainty at $30 \mathrm{AU}$ for $\mathrm{T}_{\text {obs }}=5 \times 10^{4} \mathrm{~s}$ and one of the lowest at 1 AU. It also shows one of the lowest velocity uncertainties. The two position uncertainty components, the total uncertainty and how these vary with distance from the SSB are shown in Fig. 8. For a typical distance of Mars from the SSB, 1.5 AU, the total uncertainty is $\sim 30 \mathrm{~km}$ after observing each pulsar for $5 \times 10^{4} \mathrm{~s}$. The set PSR B1937+21, B1821-24 and J1012+5307 would show a similar performance for up to 3 months. A disadvantage of using the set PSR B1937+ $21, \mathrm{~B} 0531+21$ and J0437-4715 would be the need for much higher timing model updates, for example daily, to be transmitted to the spacecraft using the ESTRACK or the DSN systems due to the much higher timing noise and rate of glitches [50] exhibited by the Crab pulsar. The two former three-pulsar sets would require much less frequent timing model updates and allow a higher level of spacecraft autonomy.

Apart from for the cases when the Crab pulsar is used, it is found that the uncertainties would not in general be expected to increase significantly above those given in Table 6 for several months without a timing model update i.e. 6 months. However, some caution is required as there is a small probability of a glitch [50] occurring in one of the pulsars and this may result in the accuracy of the timing model very rapidly degrading. No glitches have so far been observed in any MSP apart from PSR B1821-24 for which a micro-glitch has once been observed [15]. This glitch was two orders of magnitude smaller than any ever observed before. If this pulsar exhibited a similar sized glitch again during a 3 month period where there was no communication with Earth to update the timing model, the spacecraft position uncertainties for the three pulsar set PSR B1937+21, B1821-24, J04374715 would be greater by up to $\sim 10 \mathrm{~km}$. This would correspond to a total uncertainty of up to $\sim 40 \mathrm{~km}$ for an Earth-Mars trajectory, compared to $\sim 30 \mathrm{~km}$ in the more likely case of a glitch not having occurred. To mitigate the impact of a glitch in PSR B1821-24, observations could be periodically complemented by 


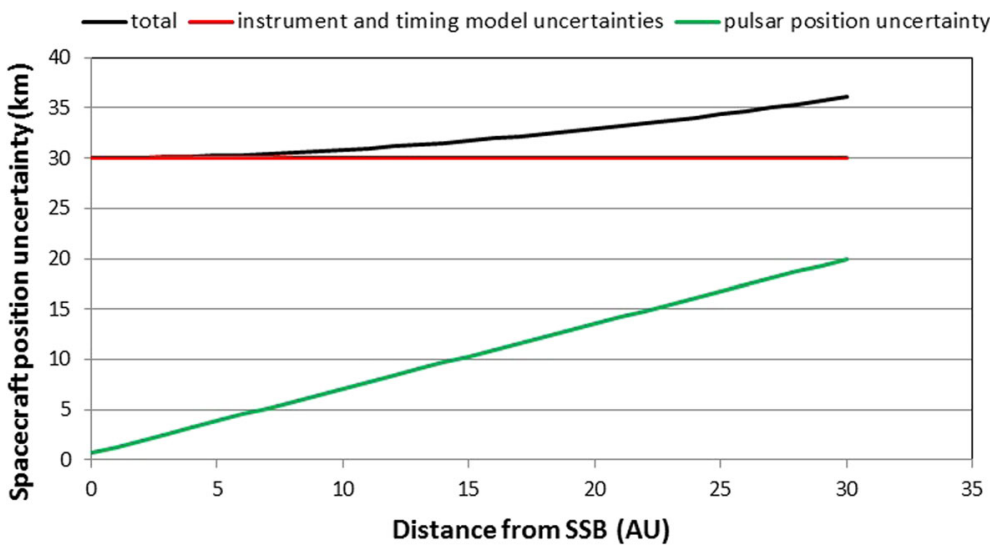

Fig. 8 The variation of the spacecraft position uncertainty and its main components with distance from the SSB for the three-pulsar set PSR B1937+21, B1821-24 and J0437-4715 and for $\mathrm{T}_{\mathrm{obs}}=5 \times 10^{4} \mathrm{~s}$. The uncertainty components shown are firstly due to the X-ray instrument and timing model uncertainties and secondly due to the pulsar position uncertainty

observations of a three pulsar set that excludes the above pulsar for crossverification and replaced by these if necessary.

In the case of using a single pulsar, uncertainties of $\sim 5$ and $\sim 1.5 \mathrm{~km}$ may be achieved in the direction of PSR B1937 +21 with $\mathrm{T}_{\text {obs }}=5 \times 10^{3} \mathrm{~s}(\sim 1 \mathrm{~h})$ and $5 \times 10^{4} \mathrm{~s}(\sim 10 \mathrm{~h})$ respectively for a distance of up to $30 \mathrm{AU}$ from the SSB. Current systems would require up to $8 \mathrm{~h}$ for providing a spacecraft with position information in this scenario. The positioning uncertainty of the DSN and ESTRACK systems in the plane of the sky are 4.5 and $45 \mathrm{~km}$ respectively for a spacecraft at $30 \mathrm{AU}$. XNAV has the potential to provide more accurate position information along the direction of PSR B1937+21. Depending on the geometry of the particular scenario, this could then be combined with the information from the DSN to enable reduced position uncertainties in the plane of the sky [34].

By assuming that the motion of the spacecraft can be adequately accounted for, the XNAV uncertainties would reduce by $\sqrt{N}$ where $N$ is the number of observations. However, in practice this will be limited by the errors in the trajectory models including due to the effects of tertiary bodies in the Solar System and solar radiation pressure over long time intervals [21].

As described in Sections 2 and 4, an XNAV system capable of simultaneous observations of a minimum of three or four pulsars for absolute navigation would provide the greatest capability. However, as described in Section 5.3.1, the implementation of an instrument allowing adequate measurement uncertainties on a spacecraft would be a significant challenge. It may be more realistic to consider an instrument making sequential observations of multiple pulsars. For this scenario, the observation times, $\mathrm{T}_{\mathrm{obs}}$, given in Section 4 and Appendix B for absolute navigation using three and four pulsars would need to be multiplied by three and four respectively.

It is found that similar position and velocity uncertainties can be achieved using three pulsars together with an accurate atomic clock instead of using four pulsars. Having to observe only three pulsars may mean a simpler instrument design is possible. Another advantage is that, obviously, if a single telescope instrument is used (see Section 5.3.1) it would take less time to sequentially observe three pulsars than four 
pulsars. The clock would need to provide suitable traceability to terrestrial time scales over the lifetime of the mission. Alternatively, this could be achieved by regular measurements with the DSN, although this would reduce spacecraft autonomy.

Pulsar position geometry is also a factor in navigation performance. As described in Appendix B, Geometric Dilution of Precision (GDOP) provides a measure of the geometry of the distribution of pulsars in the sky. Ideally the pulsars should be as widely distributed in the sky as possible.

It should be emphasised that the uncertainties derived in this paper correspond to those for the Wolter-I 'improved PSF' focussing instrument as described in Section 5 with the aim of achieving a realistic spacecraft subsystem. These will differ from those of other X-ray instruments.

The instrumentation needs of an XNAV system have been shown in Section 5 to be compatible with available technologies. However, significant improvements in performance and increased spacecraft autonomy are expected from the adoption of new technology in the next decade. Potential technological improvements exist in all three of the major subsystems considered here as follows. Improving the optic reduces background noise and offers lower positioning uncertainty. Improving the detector system e.g. to generate very high time resolution would lead to better absolute timing of a pulsar and allow improved estimates of a pulse TOA. A time resolution requirement of $<1 \mu$ s may be met with existing technology, but the significant performance improvement offered by a 100 ns uncertainty may be achieved in the next decade. Finally, improvements in the stability of atomic clocks for space applications is also expected and this would offer the possibility of reducing the number of pulsars that need to be observed to allow autonomous navigation.

Radio observations of pulsars could in principle be used in combination with or as an alternative to X-ray. However, the potential requirement of large radio antennas [8] means that a practical solution would need to be identified to meet the resource constraints of a deep space mission.

\section{Conclusions}

In this paper we have investigated the feasibility of deep-space navigation using X-ray pulsars. Simulations show that the pulsar PSR B1937+21 has potential to allow spacecraft positioning uncertainties of $\sim 2$ and $\sim 5 \mathrm{~km}$ in the direction of the pulsar after observation times of 10 and $1 \mathrm{~h}$ respectively, for distances up to $30 \mathrm{AU}$. The advantage of this technique is that, unlike existing systems such as NASA's DSN and ESA's ESTRACK networks, this could be achieved autonomously on-board the spacecraft, using an X-ray instrument of effective area $\sim 50 \mathrm{~cm}^{2}$ and a high performance atomic clock. A practical X-ray telescope may, for example, be an 'improved PSF' version of the BepiColombo-MIXS instrument. For a manned mission to Mars, where an XNAV system could provide valuable redundancy, observations of the three pulsars PSR B1937+21, B1821-24 and J0437-4715, would enable a three-dimensional positioning uncertainty of $\sim 30 \mathrm{~km}$ for up to 3 months without having to contact Earth-based systems. This would require a total observation time of $10 \mathrm{~h}$ if using an instrument with multiple telescopes or 2 days with a single telescope. The uncertainty may be lower using longer observations, although at greater ranges such as $30 \mathrm{AU}$ the present 
limiting factor is often due to uncertainties in the pulsar positions on the sky. If these were to significantly reduce in future, the navigation uncertainties would also reduce in such cases.

X-ray instrumentation suitable for use in an operational XNAV subsystem must be designed to require only modest resources, especially in terms of size/volume, mass and power. In addition to our Wolter-1 (BepiColombo) concept which involves a focussing optic, the possible options for future developments in terms of simpler and lower-cost Kirkpatrick-Baez or Lobster optics have been examined. Significant scope exists to derive an optic from one of these simpler designs with better performance than currently possible. Detectors with the necessary count rate capacity, time resolution and quantum efficiency are commercially available but electronic time tagging of X-ray photons to the required accuracy needs further study. Absolute time uncertainty onboard a spacecraft is one of the limiting factors for spacecraft autonomy. This could be improved by use of a sufficiently accurate atomic clock.

Acknowledgments The work reported in this paper is based on that performed primarily under European Space Agency Contract No. 4000105938/12/NL/KML. We thank Pietro Giordano and Jaron Samson for their interest and encouragement in the study. Microchannel plate optics have been developed by Photonis SAS, Brive France, in collaboration with the University of Leicester funded by ESA contracts. We thank Paul Houghton and Jon Sykes (University of Leicester) for providing the BepiColombo MIXS-T diagram in Fig. 6. We are grateful to John Davis at the National Physical Laboratory for constructive comments during preparation of the manuscript.

\section{Appendix}

\section{Appendix A: estimation of range error contribution due to the instrument, using an analytic formula}

From the basic statistics of a frequency distribution it follows that (e.g. [62, 69]; see also [48], Sect. 8.1.2):

$$
\sigma_{\mathrm{L}} \sim c \sigma_{\mathrm{TOA}} \sim \mathrm{K} c \mathrm{HWHM} \text { pulse } / \mathrm{SNR}
$$

where $\sigma_{\mathrm{L}}$ is the spacecraft range error; $c$ is the speed of light; $\sigma_{\mathrm{TOA}}$ is the pulse TOA error; $\mathrm{HWHM}_{\text {pulse }}$ is the pulse 'characteristic width' expressed as Half Width at Half Maximum (HWHM); SNR is the Signal-to-Noise Ratio; $\mathrm{K}$ is a dimensionless factor which embodies the detailed shape of the profile representing a weighting of the SNR across the profile with a typical value of between 1 and 0.5. $\sigma_{\mathrm{L}}$ and $\sigma_{\mathrm{TOA}}$ can then be expressed in terms of the characteristics of the pulsar and the observing instrument, as detailed below.

SNR can be expressed as:

$$
\mathrm{SNR}=\mathrm{C}_{\mathrm{p}} /\left(\mathrm{C}_{\mathrm{p}}+\mathrm{C}_{\mathrm{s}}+\mathrm{C}_{\mathrm{b}}\right)^{0.5}
$$

where: $C_{p}=$ net pulsed counts summed over the pulse profile; $C_{s}=$ unpulsed source counts; $\mathrm{C}_{\mathrm{b}}=$ counts from all other background contributions within the 'source 
beam' $=\mathrm{C}_{\mathrm{bX}}+\mathrm{C}_{\mathrm{bN}} ; \mathrm{C}_{\mathrm{bX}}=$ detected counts due to "diffuse" X-ray background (XRB) from the sky; $\mathrm{C}_{\mathrm{bN}}=$ detected counts due to cosmic-ray background $(\mathrm{CRB})$ and other sources of internal detector noise. We can then derive

$$
\begin{aligned}
& \sigma_{\mathrm{L}}=c \sigma_{\mathrm{TOA}}=c \sigma_{\phi} \mathrm{P} \approx 0.5 \quad \mathrm{~K} c \mathrm{~W}_{50} \mathrm{P}\left(\mathrm{C}_{\mathrm{p}}+\mathrm{C}_{\mathrm{s}}+\mathrm{C}_{\mathrm{b}}\right)^{0.5} / \mathrm{C}_{\mathrm{p}} \\
& \sigma_{L} \approx 0.5 \mathrm{~K}_{c} \mathrm{~W}_{50} \mathrm{P}\left[\left(\mathrm{A}_{\text {eff }} \mathrm{T}_{\text {obs }} \mathrm{f}_{\text {psf }} \mathrm{F}_{\text {total }}\right)^{0.5} \mathrm{X}_{\mathrm{p}}\right]^{-1}\left[1+\left(\mathrm{f}_{\mathrm{psf}} \mathrm{F}_{\text {total }}\right)^{-1}\left(\mathrm{~F}_{\mathrm{bX}} \Omega_{\text {eff }}+\mathrm{F}_{\mathrm{bN}} \mathrm{A}_{\text {det }} / \mathrm{A}_{\text {eff }}\right)\right]^{0.5}
\end{aligned}
$$

where $\mathrm{P}$ is the pulse period (s), $\mathrm{W}_{50}$ is the pulse profile Full Width Half Maximum $[\mathrm{FWHM}]$ as a fraction of the pulse period, $\mathrm{X}_{\mathrm{p}}$ is the fraction of the power in the pulse compared to the total time-averaged flux, $A_{\text {eff }}$ is the instrument effective area $\left(\mathrm{m}^{2}\right)$ averaged over the instrument energy band, $A_{\text {det }}$ is the source-detection area $\left(\mathrm{m}^{2}\right)$ equal to the full detector area for the collimated case and the source-detection cell area for the focussing case, $\Omega_{\text {eff }}$ is the source-detection solid angle (sr) equal to the collimator FOV for the collimated case, $\mathrm{T}_{\mathrm{obs}}$ is the observation duration $(\mathrm{s}), \mathrm{f}_{\mathrm{psf}}$ is the fraction of PSF within the source-detection area, $\mathrm{F}_{\text {total }}$ is the total $\mathrm{X}$-ray flux from the pulsar, $\mathrm{F}_{\mathrm{bX}}$ is the diffuse X-ray background flux from the sky $\left(\mathrm{ph} \mathrm{cm}^{-2} \mathrm{~s}^{-1} \mathrm{sr}^{-1}\right.$ ) and $\mathrm{F}_{\mathrm{bN}}$ is the flux due to the cosmic-ray background and other sources of internal detector noise $\left(\mathrm{ct} \mathrm{cm}^{-2} \mathrm{~s}^{-1}\right.$ ). This expression for the range error comprises a term (i) $\left.0.5 \mathrm{~K} \ldots \mathrm{X}_{\mathrm{p}}\right]^{-1}$ representing the pulsar properties and a dimensionless multiplicative term (ii) $\left.\left[1+\ldots \mathrm{F}_{\mathrm{bN}} \mathrm{A}_{\mathrm{det}} / \mathrm{A}_{\mathrm{eff}}\right)\right]^{0.5}$ representing the background components. Note that the above formulation relates to the Poisson noise only, and does not include, for example, any pulse jitter noise or the effect of pulsar position uncertainty.

\section{Appendix B: Method of estimating navigation and timing uncertainties from simulations}

\section{B.1 Absolute Navigation}

As described in Section 2.2.2, absolute navigation requires three or more pulsars. The approach described in Graven et al. [34] for deriving spacecraft position error when using three pulsars can be used as a starting point. Here the main errors are due to the pulse TOA measurement and the sky positions of the pulsars. The geometry of pulsars in the sky is also an important factor. It is assumed that there is sufficently accurate traceability to terrestrial time scales on-board the spacecraft. Using an ecliptic coordinate system in cartesian form with its origin at the SSB, the spacecraft $x, y$ and $z$ position coordinates are then given by

$$
\left[\begin{array}{l}
x \\
y \\
z
\end{array}\right]=\left[\begin{array}{lll}
x_{1} & y_{1} & z_{1} \\
x_{2} & y_{2} & z_{2} \\
x_{3} & y_{3} & z_{3}
\end{array}\right]^{-1}\left[\begin{array}{l}
\frac{c P_{1}}{2 \pi} \varphi_{1} \\
\frac{c P_{2}}{2 \pi} \varphi_{2} \\
\frac{c P_{3}}{2 \pi} \varphi_{3}
\end{array}\right]
$$

where $\varphi_{i},\left[x_{i} y_{i} z_{i}\right]$ and $P_{i}$ are the measured pulse phase in radians, the unit vector and the period of the $i$ th pulsar respectively. Using the approach given in Graven et al. [34], 
which involves propagation of errors in the small perturbation case, the error in the spacecraft position is given by

$$
\left[\begin{array}{l}
\delta x \\
\delta y \\
\delta z
\end{array}\right]=\left[\begin{array}{lll}
x_{1} & y_{1} & z_{1} \\
x_{2} & y_{2} & z_{2} \\
x_{3} & y_{3} & z_{3}
\end{array}\right]^{-1}\left[\begin{array}{l}
\frac{c P_{1}}{2 \pi} \Delta \varphi_{1} \\
\frac{c P_{2}}{2 \pi} \Delta \varphi_{2} \\
\frac{c P_{3}}{2 \pi} \Delta \varphi_{3}
\end{array}\right]-\left[\begin{array}{lll}
x_{1} & y_{1} & z_{1} \\
x_{2} & y_{2} & z_{2} \\
x_{3} & y_{3} & z_{3}
\end{array}\right]^{-1}\left[\begin{array}{ccc}
\Delta x_{1} & \Delta y_{1} & \Delta z_{1} \\
\Delta x_{2} & \Delta y_{2} & \Delta z_{2} \\
\Delta x_{3} & \Delta y_{3} & \Delta z_{3}
\end{array}\right]\left[\begin{array}{l}
x \\
y \\
z
\end{array}\right]
$$

where $\Delta \varphi_{i}$ and $\left[\Delta x_{i} \Delta y_{i} \Delta z_{i}\right.$ ] are the errors in the phase measurement and the unit vector of the $i$ th pulsar respectively. The position of each pulsar $\left[x_{i} y_{i} z_{i}\right]$ is obtained from its ecliptic coordinates and $\left[\Delta x_{i} \Delta y_{i} \Delta z_{i}\right]$ from the errors in the coordinates. The error in the measured phase $\Delta \varphi_{i}$ is mainly a result of the phase measurement error due to the X-ray instrument. As decribed later, we also include in $\Delta \varphi_{i}$ a contribution for the pulsar timing model error allowing also for an estimated level of timing noise. $c P_{i} \Delta \varphi_{i} / 2 \pi$ is equivalent to the overall range error taken for the $i$ th pulsar. We assume any system level timing errors to be small compared to the error sources described above. The errors in ecliptic coordinates are derived using the errors in right ascension and declination.

The approach given in Bernhardt et al. [10] for determining spacecraft position error using three pulsars does not appear to consider the effect of pulsar position errors. This may be a suitable approximation for inner Solar System locations where the effect is relatively small, but its impact becomes increasingly important with range from the SSB [34]. Consequently, the approach used in this paper (Eq. B.2) would lead to a higher spacecraft position error than that of Bernhardt et al. [10] if the same properties of the pulsars and instrument were used, although the difference may only be significant at larger ranges.

We extend the above approach of Graven et al. [34] to the error in spacecraft velocity. It can be shown that if the velocity components of the spacecraft are given by $\dot{x}, \dot{y}$ and $\dot{z}$, the velocity error components $\delta \dot{x}, \delta \dot{y}$ and $\delta \dot{z}$ can be determined using

$$
\left[\begin{array}{l}
\delta \dot{x} \\
\delta \dot{y} \\
\delta \dot{z}
\end{array}\right]=\left[\begin{array}{lll}
x_{1} & y_{1} & z_{1} \\
x_{2} & y_{2} & z_{2} \\
x_{3} & y_{3} & z_{3}
\end{array}\right]^{-1}\left[\begin{array}{l}
\Delta d_{1} \\
\Delta d_{2} \\
\Delta d_{3}
\end{array}\right]-\left[\begin{array}{lll}
x_{1} & y_{1} & z_{1} \\
x_{2} & y_{2} & z_{2} \\
x_{3} & y_{3} & z_{3}
\end{array}\right]^{-1}\left[\begin{array}{ccc}
\Delta x_{1} & \Delta y_{1} & \Delta z_{1} \\
\Delta x_{2} & \Delta y_{2} & \Delta z_{2} \\
\Delta x_{3} & \Delta y_{3} & \Delta z_{3}
\end{array}\right]\left[\begin{array}{c}
\dot{x} \\
\dot{y} \\
\dot{z}
\end{array}\right]
$$

where $\Delta d_{i}$ is the error in $d_{i}$ given by

$$
d_{i}=\frac{c\left(f_{i}-f_{m i}\right)}{f_{m i}}
$$

where $f_{i}$ is the measured pulse frequency of the $i$ th pulsar, and $f_{m i}$ is the expected pulse frequency in the reference frame of the SSB, as given by the pulsar's timing model. 
In the case where there is a significant spacecraft clock time-offset from terrestrial time scales, a minimum of four pulsars is required in order to also estimate this offset. We extend the above approach to the four pulsar case where the position and time errors are given by

$$
\left[\begin{array}{l}
\delta x \\
\delta y \\
\delta z \\
-c \delta t_{c}
\end{array}\right]=\left[\begin{array}{llll}
x_{1} & y_{1} & z_{1} & 1 \\
x_{2} & y_{2} & z_{2} & 1 \\
x_{3} & y_{3} & z_{3} & 1 \\
x_{4} & y_{4} & z_{4} & 1
\end{array}\right]^{-1}\left[\begin{array}{l}
\frac{c P_{1}}{2 \pi} \Delta \varphi_{1} \\
\frac{c P_{2}}{2 \pi} \Delta \varphi_{2} \\
\frac{c P_{3}}{2 \pi} \Delta \varphi_{3} \\
\frac{c P_{4}}{2 \pi} \Delta \varphi_{4}
\end{array}\right]-\left[\begin{array}{llll}
x_{1} & y_{1} & z_{1} & 1 \\
x_{2} & y_{2} & z_{2} & 1 \\
x_{3} & y_{3} & z_{3} & 1 \\
x_{4} & y_{4} & z_{4} & 1
\end{array}\right]^{-1}\left[\begin{array}{lll}
\Delta x_{1} & \Delta y_{1} & \Delta z_{1} \\
\Delta x_{2} & \Delta y_{2} & \Delta z_{2} \\
\Delta x_{3} & \Delta y_{3} & \Delta z_{3} \\
\Delta x_{4} & \Delta y_{4} & \Delta z_{4}
\end{array}\right]\left[\begin{array}{l}
x \\
y \\
z
\end{array}\right](\mathrm{B} .5)
$$

where $\delta t_{c}$ is the error in the clock time-offset. This is analogous to pseudorange errors determined in GPS [44]. In this case, the velocity error components $\delta \dot{x}, \delta \dot{y}$ and $\delta \dot{z}$ can be determined using

$$
\left[\begin{array}{l}
\delta \dot{x} \\
\delta \dot{y} \\
\delta \dot{z} \\
-c \delta \dot{t}_{c}
\end{array}\right]=\left[\begin{array}{llll}
x_{1} & y_{1} & z_{1} & 1 \\
x_{2} & y_{2} & z_{2} & 1 \\
x_{3} & y_{3} & z_{3} & 1 \\
x_{4} & y_{4} & z_{4} & 1
\end{array}\right]^{-1}\left[\begin{array}{l}
\Delta d_{1} \\
\Delta d_{2} \\
\Delta d_{3} \\
\Delta d_{4}
\end{array}\right]-\left[\begin{array}{llll}
x_{1} & y_{1} & z_{1} & 1 \\
x_{2} & y_{2} & z_{2} & 1 \\
x_{3} & y_{3} & z_{3} & 1 \\
x_{4} & y_{4} & z_{4} & 1
\end{array}\right]^{-1}\left[\begin{array}{lll}
\Delta x_{1} & \Delta y_{1} & \Delta z_{1} \\
\Delta x_{2} & \Delta y_{2} & \Delta z_{2} \\
\Delta x_{3} & \Delta y_{3} & \Delta z_{3} \\
\Delta x_{4} & \Delta y_{4} & \Delta z_{4}
\end{array}\right]\left[\begin{array}{l}
\dot{x} \\
\dot{y} \\
\dot{z}
\end{array}\right],
$$

where $\delta \dot{t}_{c}$ is the error in the drift rate of the clock.

Equation B.2 enables simulation of errors for position, using errors in pulse phase measurements and pulsar positions for three pulsars. Similarly Eq. B.5 enables simulation of errors for position and time, using the errors for four pulsars. Phase measurement errors of the instrument $\left(=\sigma_{\mathrm{TOA}, \mathrm{i}} / \mathrm{P}_{\mathrm{i}}\right)$ due to combined source- and backgroundnoise contributions for both focussing and collimated instruments have generally been estimated analytically as described in Section 3.3.1 and Appendix A. However, for the five pulsars PSR B1937+21, B1821-24, J1012+5307, J0437-4715 and B0531+21 these have been obtained using simulations of pulse profiles as described in Section 3.3.2. Position and time errors have been simulated by conducting Monte Carlo simulations using the errors in the pulse phase measurements and pulsar positions. Due to the large numbers of three- and four-pulsar combinations that are possible using 35 pulsars, we have reduced the amount of computation by simulating errors for a smaller sample of 10 pulsars with the lowest values of range error contribution due to the instrument as given in Table 1. Furthermore, as can be seen from Table 1, a focussing instrument enables significantly lower range errors than a collimator. Consequently, we present the results of simulations considering a focussing instrument for the 10 pulsars with the lowest range errors using such an instrument.

The pulsar positions, pulsar frequencies and their errors have been taken from the Xray pulsar catalogue described in Section 3. Random errors, according to a Gaussian distribution with 1 sigma given by the uncertainty in the relevant parameter, have been generated for each pulsar as inputs to Eq. B. 2 in order to determine the $\delta x, \delta y$ and $\delta z$ error components. This is repeated 100 times in order that the distribution of output 
errors in each case can be used to estimate a representative value for the 1-sigma position uncertainty. A similar process is used to derive 1-sigma uncertainties in all other PVT estimation cases. The instrument effective area is based on existing technology, i.e. the BepiColombo MIXS-T instrument [29] which has an effective area of $0.005 \mathrm{~m}^{2}$, as described in Section 5 .

The ATNF pulsar catalogue [52] gives a value for the uncertainty in the Crab pulsar timing model frequency of $10^{-7} \mathrm{~Hz}$. This has been derived using a long data-set of several years [49] and is affected at least by timing noise in the rotation of the pulsar. We consider that this value may be pessimistic and have used a value of $10^{-10} \mathrm{~Hz}$ that we believe should be realistic using a shorter span of Crab pulsar data.

Table 8 shows the six three-pulsar sets that give the lowest position uncertainties for a spacecraft located at a distance of 30 AU from the SSB at ecliptic coordinates given by $x=30 \mathrm{AU}, y=0 \mathrm{AU}$ and $z=0 \mathrm{AU}$. The position uncertainty given in each case is obtained from the square-root of the sum of the squares of the uncertainties along each of the $x$-, $y$ - and $z$-axes of the ecliptic coordinate system which form an error ellipsoid. The observation time for each pulsar is taken to be $5 \times 10^{4} \mathrm{~s}$, resulting in a time-area product of $250 \mathrm{~m}^{2} \mathrm{~s}$. It can be seen that the pulsar-set given by B1937+21, B1821-24 and J0437-4715 provides the lowest uncertainties. Pulsar position geometry is also a factor in navigation performance. A measure of the geometry in this case is the Position Dilution of Precision (PDOP) [44, 59]. A lower PDOP value corresponds to a better geometry. The value of this is also shown for each pulsar-set in Table 8. Similarly, Table 9 shows the five four-pulsar-sets that give the lowest combined position and clock time-offset uncertainties for a spacecraft located at the same point in the Solar System as above. The best set corresponds to B1937+21, B1821-24, J0437-4715 and $\mathrm{J} 1012+53$. The pulsar position geometry factor in this case, for position and time estimation, is called the Geometric Dilution of Precision (GDOP) and is also given in Table 9 .

For some pulsars, a representative value has also been taken for the pulsar timing model error. This was the case for the pulsars PSR B1937+21, B1821-24, J1012+ $5307, \mathrm{~J} 0437-4715$ and B0531 +21. For the first four pulsars we have assumed a latency period of 3 months between the last TOA used to derive the timing model and the XNAV observation epoch on-board the spacecraft. With the exception of the Crab pulsar, the timing model error, allowing also for an estimate of timing noise, is expected

Table 8 Position uncertainties for absolute navigation in the ecliptic plane using three pulsars and an observation time of $5 \times 10^{4} \mathrm{~s}$ for each pulsar

\begin{tabular}{lll}
\hline Pulsar-set & PDOP & Position uncertainty $(\mathrm{km})$ \\
\hline B1937+21, B1821-24, J0437-4715 & 2.9 & 35 \\
B1937+21, B1821-24, J1012+5307 & 2.8 & 50 \\
B1821-24, J0437-4715, J0030+0451 & 1.8 & 80 \\
B1937+21, J1012+5307, J0030+0451 & 1.9 & 85 \\
J1012+5307, J0437-4715, J0030+0451 & 2.1 & 90 \\
B1937+21, B 0531+21, J0437-4715 & 2.6 & 95 \\
\hline
\end{tabular}

These apply to a spacecraft with ecliptic coordinates $x=30$ AU, $y=0$ AU and $z=0$ AU. The six pulsar-sets with the lowest uncertainties are shown together with the PDOP in each case. 
Table 9 Position and clock time-offset uncertainties for absolute navigation in the ecliptic plane using four pulsars and an observation time of $5 \times 10^{4} \mathrm{~s}$ for each pulsar

\begin{tabular}{llll}
\hline Pulsar-set & GDOP & $\begin{array}{l}\text { Time-offset uncertainty } \\
(\mathrm{s})\end{array}$ & $\begin{array}{l}\text { Position uncertainty } \\
(\mathrm{km})\end{array}$ \\
\hline $\begin{array}{l}\text { B1937+21, B1821-24, J1012+5307, PJ0437- } \\
\quad 4715\end{array}$ & 2.6 & 0.00004 & 35 \\
$\begin{array}{l}\text { B1937+21, B1821-24, J1012+5307, } \\
\quad \text { J0030+0451 }\end{array}$ & 2.5 & 0.0001 & 65 \\
$\begin{array}{l}\text { B1821-24, J1012+5307, J0437-4715, } \\
\quad \text { J0030+0451 }\end{array}$ & 1.7 & 0.00005 & 75 \\
$\begin{array}{l}\text { B1937+21, 0531+21, B1821-24, J1012+5307 } \\
\text { B1937+21, J1012+5307, J0437-4715, }\end{array}$ & 2.5 & 0.0001 & 85 \\
$\quad$ J0030+0451 & 2.2 & 0.00005 & 100 \\
\hline
\end{tabular}

These apply to a spacecraft with ecliptic coordinates $x=30 \mathrm{AU}, y=0 \mathrm{AU}$ and $z=0 \mathrm{AU}$. The five pulsar-sets with the lowest position uncertainties are shown. Values of GDOP are also shown for each pulsar-set

to be highest as a fraction of the TOA measurement error (obtained using simulations of pulse profiles as in Section 3.3.2) for PSR B1821-24. Based on the data presented in Cognard et al. [17], we have taken a first order representative value of this to be $5 \mu \mathrm{s}$, equivalent to $\sim 50 \%$ of the TOA measurement error for an observation time of $5 \times 10^{4}$ s. For PSR B1937+21, J1012 +5307 and J0437-4715 this is taken to be of order $20 \%, 20 \%$ and $1 \%$ respectively based on the data given in Cognard et al. [16], Lange et al. [46] and Verbiest et al. [75]. In the case of the Crab pulsar, the timing model error is likely to be at least tens of microseconds for a latency period which could conceivably be 1 month or less due to timing models for this pulsar being published more frequently compared to those of other pulsars [49]. We have assumed the error to be $10 \mu \mathrm{s}$ which corresponds to a latency period of order 3 days. For cases where this is below $\sim 200 \mu$ s, the dominant error source would be that due to the pulsar's position error.

The first five rows of Table 10 show the five three-pulsar-sets that offer the lowest velocity uncertainties for a time-area product of $250 \mathrm{~m}^{2} \mathrm{~s}$ based on the 10 pulsars assessed in detail. In this case the spacecraft is considered as having a velocity of 30 $\mathrm{kms}^{-1}$ relative to the SSB in the direction of zero ecliptic longitude and latitude. The

Table 10 Velocity uncertainties for absolute navigation in the ecliptic plane using three pulsars and an observation time of $5 \times 10^{4} \mathrm{~s}$ for each pulsar

\begin{tabular}{ll}
\hline Pulsar-set & Velocity uncertainty $\left(\mathrm{ms}^{-1}\right)$ \\
\hline B1937+21, B1821-24, J0218+4232 & 0.4 \\
B1821-24, J0218+4232, J0437-4715 & 0.5 \\
B1937+21, J0218+4232, J0437-4715 & 0.6 \\
B0531+21, B J0218+4232, J0437-4715 & 0.6 \\
B1937+21, B 0531+21, J0437-4715 & 0.6 \\
B1937+21, B1821-24, J0437-4715 & 0.6 \\
\hline
\end{tabular}

The six pulsar-sets with the lowest uncertainties are shown based on the 10 pulsars assessed. 
five pulsar-sets do not include the pulsar-set identified with the lowest position uncertainty in Table 8. The velocity uncertainty for this is shown for comparison in the bottom row of Table 10. Similarly, Table 11 shows four-pulsar-sets with their velocity uncertainties.

\section{B.2 Delta-correction measurement using a single pulsar}

This is the simplest strategy [34, 69] as described in Section 2.2.1 and represented in Fig. 1. The position-offset $\underline{\Delta r}$ can be defined as follows

$$
\underline{\Delta r}=\underline{r}-\underline{\tilde{r}},
$$

where $\underline{r}$ is the actual position of the spacecraft and $\underline{\tilde{r}}$ is the estimated position. Using a similar approach to that used for absolute navigation (see Section B.1), the error in the position-offset in the direction of the pulsar is given by

$$
\underline{\hat{n}} \cdot \delta \underline{\Delta r}=\frac{c P}{2 \pi} \Delta \varphi-d \underline{\hat{n}} \cdot \underline{r}
$$

where $d \underline{\hat{n}}$ is the error in the unit vector to the pulsar, $\Delta \varphi$ is the error in the pulse phase measurement due to the X-ray instrument and pulsar timing model. Equation B.8 has been used to simulate uncertainties in spacecraft position-offset. The simulation assumes a spacecraft position in the ecliptic plane with a range of 30 AU from the SSB at ecliptic coordinates given by $x=30 \mathrm{AU}, y=0 \mathrm{AU}$ and $z=0$ AU. Table 12 shows the six pulsars that give the lowest position uncertainties in the direction of the pulsar with a focussing instrument and a time-area product of $250 \mathrm{~m}^{2} \mathrm{~s}$. PSR B1937+21 yielded the lowest uncertainty.

Table 11 Velocity and clock time-drift rate uncertainties for absolute navigation in the ecliptic plane using

\begin{tabular}{|c|c|c|}
\hline Pulsar-set & $\begin{array}{l}\text { Time-drift rate uncertainty } \\
\left(\mathrm{ss}^{-1}\right)\end{array}$ & $\begin{array}{l}\text { Velocity uncertainty } \\
\left(\mathrm{ms}^{-1}\right)\end{array}$ \\
\hline $\mathrm{B} 1937+21, \mathrm{~B} 0531+21, \mathrm{~B} 1821-24, \mathrm{~J} 0218+4232$ & $0.2 \times 10^{-9}$ & 0.4 \\
\hline $\begin{array}{l}\text { B1937+21, B0531+21, J0218+4232, J0437- } \\
\quad 4715\end{array}$ & $0.3 \times 10^{-9}$ & 0.4 \\
\hline $\begin{array}{l}\text { B0531+21, B1821-24, J0218+4232, J0437- } \\
\quad 4715,\end{array}$ & $0.3 \times 10^{-9}$ & 0.5 \\
\hline $\begin{array}{l}\mathrm{B} 1937+21, \mathrm{~B} 1821-24, \mathrm{~J} 0218+4232, \\
\quad \mathrm{~J} 1012+5307\end{array}$ & $0.6 \times 10^{-9}$ & 0.6 \\
\hline $\begin{array}{l}\text { B1937+21, B1821-24, J1012+5307, J0437- } \\
\quad 4715\end{array}$ & $0.8 \times 10^{-9}$ & 0.6 \\
\hline $\mathrm{B} 1937+21, \mathrm{~B} 0531+21, \mathrm{~B} 1821-24, \mathrm{~J} 1012+5307$ & $0.3 \times 10^{-9}$ & 0.7 \\
\hline
\end{tabular}
four pulsars and an observation time of $5 \times 10^{4} \mathrm{~s}$ for each pulsar

The six pulsar-sets with the lowest velocity uncertainties are shown based on the 10 pulsars assessed. The last row shows the uncertainty for the only pulsar set given in Table 9 which includes the Crab pulsar. This corresponds to the pulsar set with the 8th lowest uncertainty 
Table 12 Position uncertainties in the ecliptic plane using the delta-correction method with a single pulsar and an observation time of $5 \times 10^{4} \mathrm{~s}$

\begin{tabular}{ll}
\hline PSR & Position uncertainty in the direction of the pulsar $(\mathrm{km})$ \\
\hline B1937+21 & 1.5 \\
B1821-24 & 12 \\
J0437-4715 & 16 \\
J1012+5307 & 30 \\
J0030+0451 & 65 \\
B0531+21 & 70 \\
\hline
\end{tabular}

These apply to a spacecraft with ecliptic coordinates $x=30 \mathrm{AU}, y=0 \mathrm{AU}$ and $z=0 \mathrm{AU}$. The six pulsars with the lowest uncertainties are shown

Similarly to above, the velocity-offset of the spacecraft, $\underline{\Delta v}$, can be defined as

$$
\underline{\Delta v}=\underline{v}-\underline{\tilde{v}}
$$

where, $\underline{v}$, is the actual velocity of the spacecraft and $\underline{\tilde{v}}$ is the estimated velocity. The error in the velocity-offset, $\delta \underline{\Delta v}$, in the direction to the pulsar is given by

$$
\underline{\hat{n}} \cdot \delta \underline{\Delta v}=\Delta d-d \underline{\hat{n}} \cdot \underline{v}
$$

where $d \underline{\hat{n}}$ is as described above and $\Delta d$ is the error in $d$, as defined in Eq. B.4, and given by the error in the pulse frequency measurement due to the $\mathrm{X}$-ray instrument and the frequency error of the pulsar timing model. We assume any system level timing errors to be small compared to the error sources described above. Table 13 shows the seven pulsars that give the lowest velocity uncertainties in the direction of the pulsar with a focussing instrument and a time-area product of $250 \mathrm{~m}^{2} \mathrm{~s}$. As in the previous cases, the spacecraft is considered as having a velocity of $30 \mathrm{kms}^{-1}$ relative to the SSB in the direction of zero degrees ecliptic longitude and latitude. In this case, PSR B1937 + 21 again yielded the lowest uncertainty.

Table 13 Velocity uncertainties in the ecliptic plane using the delta-correction method with a single pulsar and an observation time of $5 \times 10^{4} \mathrm{~s}$

\begin{tabular}{lll}
\hline PSR & Velocity uncertainty in the direction of the pulsar $\left(\mathrm{ms}^{-1}\right)$ & Timing model frequency error $(\mathrm{Hz})$ \\
\hline $\mathrm{B} 1937+21$ & 0.03 & $1.3 \times 10^{-12}$ \\
$\mathrm{~J} 0218+4232$ & 0.06 & $3 \times 10^{-10}$ \\
$\mathrm{~B} 1821-24$ & 0.07 & $6 \times 10^{-10}$ \\
$\mathrm{~B} 0531+21$ & 0.07 & $10^{-10}$ \\
$\mathrm{~J} 0205+6449$ & 0.30 & $2 \times 10^{-11}$ \\
$\mathrm{~J} 0437-4715$ & 0.35 & $3 \times 10^{-12}$ \\
$\mathrm{~J} 1012+5307$ & 0.60 & $4 \times 10^{-12}$ \\
\hline
\end{tabular}

The seven pulsars with the lowest uncertainties are shown based on the 10 pulars assessed. The timing model frequency error used is also given in each case 
Open Access This article is distributed under the terms of the Creative Commons Attribution 4.0 International License (http://creativecommons.org/licenses/by/4.0/), which permits unrestricted use, distribution, and reproduction in any medium, provided you give appropriate credit to the original author(s) and the source, provide a link to the Creative Commons license, and indicate if changes were made.

\section{References}

1. Angel, J.R.P.: Lobster eyes as X-ray telescopes. Astrophys. J. 233, 364-373 (1979)

2. Becker, W., Truemper, J.: ROSAT observations of gamma-ray pulsars: vela, PSR 1706-44 and PSR $1951+32$. Astron. Astrophys. 120C, 69 (1996)

3. Becker, W., Aschenbach, B.: X-ray Observations of Neutron Stars and Pulsars: First Results from XMMNewton, in Neutron Stars, Pulsars and Supernova Remnants, eds. W. Becker, H. Lesch, J. Truemper, MPE-Report 278, p. 64 (astro-ph/0208466) (2002)

4. Becker, W., Weisskopf, M.C., Tennant, A.F., Jessner, A., Dyks, J., Harding, A.K., Zhang, S.N.: Revealing the X-Ray emission processes of Old rotation-powered pulsars: XMM-newton observations of PSR B0950 + 08, PSR B0823 + 26, and PSR J2043 + 2740. Astrophys. J. 615, 908 (2004)

5. Becker, W., Jessner, A., Kramer, M., Testa, V., Howaldt, C.: A multiwavelength study of PSR B0628-28: the first overluminous rotation-powered pulsar? Astrophys. J. 633, 367 (2005)

6. Becker, W., Kramer, M., Jessner, A., Taam, R.E., Jia, J.J., Cheng, K.S., Mignani, R., Pellizzoni, A., De Luca, A., Slowikowska, A., Caraveo, P.A.: A multiwavelength study of the pulsar PSR B1929+10 and its X-Ray trail. Astrophys. J. 645, 1421 (2006)

7. Becker, W.: X-ray emission from pulsars and neutron stars. In: Becker, W. (ed.) Neutron stars and pulsars, pp. 91-140. Springer, ASSL (2009)

8. Becker, W., Bernhardt, M. G., Jessner, A.: Autonomous Spacecraft Navigation with Pulsars. (2013) Accessed 3 June (2013)

9. Benkhoff, J., van Casteren, J., Hayakawa, H., Fujimoto, M., Laakso, H., Novara, M., Ferri, P., Middleton, H.R., Ziethe, R.: BepiColombo-Comprehensive exploration of Mercury: Mission overview and science goals. Planet. Space Sci. 58, 2-20 (2010)

10. Bernhardt, M. G., Prinz, T., Becker, W., Walter, U.: Timing X-ray pulsars with application to spacecraft navigation. In: High-time resolution astrophysics IV. Proceedings of Science. arXiv:1011.5095v1 (2010) Accessed 23 August (2013)

11. Bloch, M., Mancini, O., McClelland, T.: Effects of radiation on performance of space-borne quartz crystal oscillators. In: Proceedings of European Frequency and Time Forum, Besancon, France, pp. 171-174, 20 24 April (2009)

12. Camilo, F., Lorimer, D.R., Bhat, N.D.R., Gotthelf, E.V., Halpern, J.P., Wang, Q.D., Lu, F.J., Mirabal, N.: Discovery of a 136 millisecond radio and X-Ray pulsar in supernova remnant G54.1 + 0.3. Astrophys. J. 574, L71-L74 (2002)

13. Chatterjee, S., Gaensler, B.M., Melatos, A., Brisken, W.F., Stappers, B.W.: Pulsed X-Ray emission from pulsar a in the double pulsar system J0737-3039. Astrophys. J. 670, 1301-1306 (2007)

14. Chester, T.J., Butman, S.A.: Navigation using X-Ray pulsars. NASA Technical Report. N81-27129, pp. 22-25. http://ipnpr.jpl.nasa.gov/progress_report/42-63/63F.PDF (1981). Accessed 23 May 2016

15. Cognard, I., Backer, D.C.: A micro-glitch in the millisecond pulsar B1821-24 in M28. Astrophys. J. 612, L125-L127 (2004)

16. Cognard, I., Bourgois, G., Lestrade, J.-F., Biraud, F., Aubry, D., Darchy, B., Drouhin, J.-P.: High-precision timing observation of the millisecond pulsar PSR 1937 + 21at nancay. Astron. Astrophys. 296, 169-179 (1995)

17. Cognard, I., Bourgois, G., Lestrade, J.-F., Biraud, F., Aubry, D., Darchy, B., Drouhin, J.-P.: High-precision timing observation of the millisecond pulsar PSR 1821-24 at nancay. Astron. Astrophys. 311, 179-188 (1996)

18. Curkendall, D. W., Border, J. S.: Delta-DOR: The one-nanoradian navigation measurement system of the deep space network - history, architecture and componentry. ResearchGate. http://www.researchgate.net/ publication/258791759_Delta-DOR_The_One-Nanoradian_Navigation_Measurement_System_of_the Deep_Space_Network___History_ĀArchitecture_and_Componentry (2013) Accessed 4 November $201 \overline{5}$

19. Cusumano, G., Hermsen, W., Kramer, M., Kuiper, L., Löhmer, O., Massaro, E., Mineo, T., Nicastro, L., Stappers, B.W.: The phase of the radio and X-ray pulses of PSR B1937 + 21. Astron. Astrophys. 410, L9 (2003) 
20. De Luca, A., Caraveo, P.A., Mereghetti, S., Negroni, M., Bignami, G.F.: On the polar caps of the three musketeers. Astrophys. J. 623, 1051-1069 (2005)

21. Deng, X.P., Hobbs, G., You, X.P., Li, M.T., Keith, M.J., Shannon, R.M., Coles, W., Manchester, R.N., Zheng, J.H., Yu, X.Z., Gao, D., Wu, X., Chen, D.: Interplanetary spacecraft navigation using pulsars. Adv Space Res. 52(9), 1602-1621 (2013)

22. Downs, G.S.: Interplanetary navigation using pulsating radio sources. NASA Technical Report. N7434150, pp. 1-12. http://ntrs.nasa.gov/archive/nasa/casi.ntrs.nasa.gov/19740026037 pdf (1974). Accessed Accessed 23 May 2016

23. Droz, F., Mosset, P., Barmaverain, G., Rochat, P., Wang, Q., Belloni, M., Mattioni, L., Schmidt, U., Pike, T., Emma, F. and Waller, P.: The on-board Galileo clocks: rubidium standard and passive hydrogen maser - current status and performance. In: Proceedings of the European Frequency and Time Forum, Braunschweig, Germany, pp. 420-426, 27-30 March (2006)

24. Emadzadeh, A. A., Speyer, J. L.: Navigation in Space by X-ray Pulsars. Springer Science \& Business Media (2011)

25. Espinoza, C.M., Lyne, A.G., Stappers, B.W., Kramer, M.: A study of 315 glitches in the rotation of 102 pulsars. Mon. Not. R. Astron. Soc. 414(2), 1679-1704 (2011)

26. Evans, I.N., Primini, F.A., Glotfelty, K.J., Anderson, C.S., Bonaventura, N.R., Chen, J.C., Davis, J.E., Doe, S.M., Evans, J.D., Fabbiano, G., Galle, E.C., Gibbs II, D.G., Grier, J.D., Hain, R.M., Hall, D.M., Harbo, P.N., He, X., Houck, J.C., Karovska, M., Kashyap, V.L., Lauer, J., McCollough, M.L., McDowell, J.C., Miller, J.B., Mitschang, A.W., Morgan, D.L., Mossman, A.E., Nichols, J.S., Nowak, M.A., Plummer, D.A., Refsdal, B.L., Rots, A.H., Siemiginowska, A., Sundheim, B.A., Tibbetts, M.S., Van Stone, D.W., Winkelman, S.L., Zografou, P.: The Chandra source catalog. Astrophys. J. 189, 37-82 (2010)

27. Feroci, M., Stella, L., van der Klis, M., et al.: The large observatory for X-ray timing (LOFT). Exp. Astron. 34, 415-444 (2012)

28. Fraser, G.W.: X-ray detectors in astronomy. Cambridge University Press, Cambridge (2009)

29. Fraser, G.W., Carpenter, J.D., Rothery, D.A., Pearson, J.F., Martindale, A., Huovelin, J., Treis, J., Anand, M., Anttila, M., Ashcroft, M., Benkoff, J., Bland, P., Bowyer, A., Bradley, A., Bridges, J., Brown, C., Bulloch, C., Bunce, E.J., Christensen, U., Evans, M., Fairbend, R., Feasey, M., Giannini, F., Hermann, S., Hesse, M., Hilchenbach, M., Jorden, T., Joy, K., Kaipiainen, M., Kitchingman, I., Lechner, P., Lutz, G., Malkki, A., Muinonen, K., Näränen, J., Portin, P., Prydderch, M., Juan, J., San Sclater, S.E., Schyns, E., Stevenson, T.J., Strüder, L., Syrjasuo, M., Talboys, D., Thomas, P., Whitford, C., Whitehead, S.: The mercury imaging X-ray spectrometer (MIXS) on BepiColombo. Planet. Space Sci 58(1-2), 79-95 (2010)

30. Galloway, D.K., Markwardt, C.B., Morgan, E.H., Chakrabarty, D., Strohmayer, T.E.: Discovery of the accretion-powered millisecond X-ray pulsar IGR J00291 + 5934. Astrophys. J. 622, L45-L48 (2005)

31. Golshan, A. R., Sheikh S. I.: On Pulse Phase Estimation and Tracking of Variable Celestial X-Ray Sources In: Institute of Navigation 63rd Annual Meeting. Cambridge, MA, April 23-25 (2007)

32. Gonzalez, M.E., Kaspi, V.M., Camilo, F., Gaensler, B.M., Pivovaroff, M.J.: Unusual pulsed X-Ray emission from the young, high magnetic field pulsar PSR J1119-6127. Astrophys. J. 630, 489-494 (2005)

33. Gotthelf, E.V., Vasisht, G., Boylan-Kolchin, M., Torii, K.: A 700 Year-old Pulsar in the Supernova Remnant Kesteven 75. Astrophys. J. 542, L37-L40 (2000)

34. Graven, P., Collins, J., Sheikh, S., Hanson, J., Ray, P., Wood, K.: XNAV for Deep Space Navigation. In: 31st Annual AAS Guidance and Control Conference. 08-054, Breckenridge CO, 4 February (2008)

35. Halpern, J.P., et al.: Neutron stars in supernova remnants. ASP Conf. Ser 271, 199 (2002)

36. Hanson, J., Sheikh, S., Graven, P., Collins, J.: Noise analysis for X-ray navigation systems. In: IEEE/ION Position, Location and Navigation Symposium. pp. 704-713, 5-8 May (2008)

37. Hessels, J.W.T., Roberts, M.S.E., Ransom, S.M., Kaspi, V.M., Romani, R.W., Ng, C.Y., Freire, P.C.C., Gaensler, B.M.: Observations of PSR J2021+3651 and its X-Ray pulsar wind nebula G75.2+0.1. Astrophys. J. 612, 389 (2004)

38. Hobbs, G.: Pulsars as gravitational wave detectors (2010) http://arxiv.org/abs/1006.3969 Accessed 4 November 2015

39. Hobbs, G., Lyne, A.G., Kramer, M.: Pulsar timing noise. Chin. J. Astron. Astrophys. 6(Suppl. 2), 169-175 (2006)

40. Hughes, J.P., Slane, P.O., Park, S., Roming, P.W.A., Burrows, D.N.: An X-Ray pulsar in the oxygen-rich supernova remnant G292.0 + 1.8. Astrophys. J. 591, L139 (2003)

41. Iess, L., Budnik, F., Colamarino, C., Corbelli, A., Di Benedetto, M., Fabbri, V., Graziani, A., Hunt, R., James, N., Lanucara, M., Maddè, R., Marabucci, M., Mariotti, G., Mercolino, M., Racioppa, P., Simone, L., Tortora, P., Westcott, M., Zannoni, M.: Improving tracking systems for deep space navigation. ResearchGate. http://www.researchgate.net/publication/257066682_IMPROVING_TRACKING_ SYSTEMS_FOR_DEEP_SPACE_NAVIGATION (2013) Accessed 22 October 2015 
42. Jahoda, K., Swank, J.H., Giles, A.B., Stark, M.J., Strohmayer, T., Zhang, W., Morgan, E.H.: In-orbit performance and calibration of the Rossi X-ray timing explorer (RXTE) proportional counter array (PCA). In: Proceedings of Soc. Photo-Opt. Instru. 2808, pp. 59-70 (1996)

43. Kaaret, P., Marshall, H.L., Aldcroft, T.L., Graessle, D.E., Karovska, M., Murray, S.S., Rots, A.H., Schulz, N.S., Seward, F.D.: Chandra observations of the young pulsar PSR B0540-69. Astrophys. J. 546, 1159 (2001)

44. Kaplan, E.D.: Understanding GPS Principles and Applications. Artech House (1996)

45. Kirkpatrick, P., Baez, A.V.: Formation of optical images by X-rays. J. Opt. Soc. Am. 38, 766-774 (1948)

46. Lange, C., Camilo, F., Wex, N., Kramer, M., Backer, D.C., Lyne, A.G., Doroshenko, O.: Precision timing measurements of PSR J1012 + 5307. Mon. Not. R. Astron. Soc. 326, 274 (2001)

47. Lobanov, A.P., Horns, D., Muxlow, T.W.B.: VLBI imaging of a flare in the crab nebula: more than just a spot. Astron. Astrophys. 533, A10 (2011)

48. Lorimer, D., Kramer, M.: Handbook of pulsar astronomy. Cambridge University Press, Cambridge (2005)

49. Lyne, A.G., Pritchard, R.S., Graham Smith, F.: 23 years of crab pulsar rotational history. Mon. Not. R. Astron. Soc. 265, 1003-1012 (1993)

50. Lyne, A.G., Smith, F.G.: Pulsar astronomy. Cambridge University Press, Cambridge (2012)

51. Majewski, P., Andricek, L., Bahr, A., De Vita, G., Gunther, B., Hermenau, K., Hilchenbach, M., Lauf, T., Lechner, P., Lutz, G., Miessner, D., Porro, M., Reiffers, J., Richter, R., Schaller, G., Schnecke, M., Schopper, F., Soltau, H., Stefanescu, A. Strecker, R., Struder, L., Treis, J.: DEPFET macropixel detectors for MIXS: integration and qualification of the flight detectors. IEEE T. Nucl. Sci. 59, 2479-2486 (2012)

52. Manchester, R.N., Hobbs, G.B., Teoh, A., Hobbs, M.: The Australia telescope national facility pulsar catalogue. Astron. J. 129, 1993-2006 (2005). http://www.atnf.csiro.au/research/pulsar/psrcat Accessed 22 October 2015

53. Martin-Carrillo, A., Kirsch, M.G.F., Caballero, I., et al.: The relative and absolute timing accuracy of the EPIC-pn camera on XMM-newton, from X-ray pulsations of the crab and other pulsars. Astron. Astrophys. 545, A126 (2012)

54. Martindale, A., Pearson, J. F., Fraser, G. W., Carpenter, J. D., Willingale, R., Stevenson, T., Whitford, C., Giannini, F., Fairbend, R., Seguy, J., Sclater, E., Delgado, I., Kaipiainen, M., Nenonen, S., Pilvi, T., Schyns, E., Bulloch, C., Sawyers, C., Muinonen, K.: The Mercury Imaging X-ray Spectrometer: optics design and characterisation. In: Proceedings of Soc. Photo-Opt. Instru. 7441 (2009)

55. McGowan, K.E., Kennea, J.A., Zane, S., Córdova, F.A., Cropper, M., Ho, C., Sasseen, T., Vestrand, W.T.: Detection of pulsed X-Ray emission from XMM-newton observations of PSR J0538 + 2817. Astrophys. J. 591, 380-387 (2003)

56. McGowan, K.E., Zane, S., Cropper, M., Kennea, J.A., Córdova, F.A., Ho, C., Sasseen, T., Vestrand, W.T.: XMM-newton observations of PSR B1706-44. Astrophys. J. 600, 343-350 (2004)

57. Murray, S.S., Slane, P.O., Seward, F.D., Ransom, S.M., Gaensler, B.M.: Discovery of X-Ray pulsations from the compact central source in the supernova remnant 3C 58 astrophys. Astrophys. J. 568, 226-231 (2002)

58. Nicastro, L., Cusumano, G., Löhmer, O., Kramer, M., Kuiper, L., Hermsen, W., Mineo, T., Becker, W.: BeppoSAX observation of PSR B1937 + 21. Astron. Astrophys. 413, 1065-1072 (2004)

59. Parkinson, B. W.: GPS Error Analysis. In: Parkinson, B. W., Spilker, J.J. (eds.) Global Positioning System: Theory and Applications Volume 1, pp. 469-484. American Institute of Aeronautics, Inc. (1996)

60. Porro, M.: Development of the DEPFET sensor with signal compression: A large format X-ray imager with mega-frame readout capability for the European XFEL. Nuclear Science Symposium and Medical Imaging Conference (NSS/MIC), pp. 1424-1434 (2011)

61. Ptak, A., Griffiths, R.: XAssist: a system for the automation of X-ray astrophysics analysis in: ADASS XII. ASP Conf Ser. 295, 465 (2003)

62. Ray, P.S., Sheikh, S. I., Graven, P. H., Wolff, M. T., Wood, K. S., Gendreau.: Deep space navigation using celestial X-ray sources. In: Proceedings of the Institute of Navigation, National Technical Meeting. San Diego, CA. pp. 101-109, 28-30 January (2008)

63. Reichley, P., Downs, G., Morris, G.: Use of pulsar signals as clocks. NASA Jet Propulsion Laboratory Quarterly Technical Review. 1, 2, pp. 80-86 http://ntrs.nasa.gov/archive/nasa/casi.ntrs.nasa.gov/ 19710021630 pdf (1971). Accessed 15 May 2016

64. Roberts, S.E., Romani, R.W., Johnston, S.: Multiwavelength studies of PSR J1420-6048, a young pulsar in the kookaburra. Astrophys. J. 561, L187-L190 (2001)

65. ROSAT Consortium.: ROSAT Source Catalogues. ROSAT News. 74 (2001)

66. Rots, A.H., Jahoda, K., Macomb, D.J., Kawai, N., Saito, Y., Kaspi, V.M., Lyne, A.G., Manchester, R.N., Backer, D.C., Somer, A.L., Marsden, D., Rothschild, R.E.: ROSSI X-ray timing explorer absolute timing results for the pulsars B1821-24 and B1509-58. Astrophys. J. 501, 749-757 (1998) 
67. Schiller, S., Gorlitz, A., Nevsky, A., Alighanbari, S., Vasilyev, S., Abou-Jaoudeh, C., Mura, G., Franzen, T., Sterr, U., Falke, S., Lisdat, C., Rasel, E., Kulosa, A., Bize, S., Lodewyck, J., Tino, G. M., Poli, N., Schioppo, M., Bongs, K., Singh, Y., Gill, P., Barwood, G. P., Ovchinnikov, Y. B., Stuhler, J., Kaenders, W., Braxmaier, C., Holzwarth, R., Donati, A., Lecomte, S., Calonico, D., Levi, F.: The space optical clocks project: development of high-performance transportable and breadboard optical clocks and advanced subsystems. In: Proceedings of the European Frequency and Time Forum, Gothenburg, Sweden, pp. 412-418, 24-26 April (2012)

68. Sheikh, S. I., Golshan, A. R., Pines, D. J.: Absolute and Relative Position Determination Using Variable Celestial X-ray Sources. In: 30th Annual AAS Guidance and Control Conference. pp. 855-874, 3-7 February (2007)

69. Sheikh, S.I., Pines, D.J., Wood, K.S., Ray, P.S., Lovellette, M.N., Wolff, M.T.: Spacecraft navigation using X-ray pulsars. J. Guid. Control. Dynam. 29(1), 49-63 (2006)

70. Sheikh, S. I., Ray, P. S., Weiner, K., Wolff, M. T., Wood, K. S.: Relative Navigation of Spacecraft Utilizing Bright, Aperiodic Celestial Sources, In: Institute of Navigation 63rd Annual Meeting. Cambridge, MA, 23-25 April (2007)

71. Shemar, S.L., Lyne, A.G.: Observations of pulsar glitches. Mon. Not. R. Astron. Soc. 282, 677-690 (1996)

72. Tjoelker, R. L., Burt, E. A., Chung, S., Hamell, R. L., Prestage, J. D., Tucker, B., Cash, P., Lutwak, R.: Mercury atomic frequency standards for space based navigation and time-keeping. In: Proceedings of the 43rd Annual Precise Time and Time Interval Systems and Applications Meeting, Long Beach, California, pp. 293-304, 14-17 November (2011)

73. Torii, K., Saito, Y., Nagase, F., Yamagami, T., Kamae, T., Hirayama, M., Kawai, N., Sakurai, I., Namiki, M., Shibata, S., Gunji, S., Finley, J.P.: ASCA detection of pulsed X-Ray emission from PSR J0631 + 1036. Astrophys. J. 551, L151-L154 (2001)

74. Torii, K., Tsunemi, H., Dotani, T., Mitsuda, K., Kawai, N., Kinugasa, K., Saito, Y., Shibata, S.: Spin-down of the 65 millisecond X-Ray pulsar in the supernova remnant G11.2-0.3. Astrophys. J. 523, L69-L72 (1999)

75. Verbiest, J.P.W., Bailes, M., van Straten, W., Hobbs, G.B., Edwards, R.T., Manchester, R.N., Bhat, N.D.R., Sarkissian, J.M., Jacoby, B.A., Kulkarni, S.R.: Precision timing of PSR J0437-4715: an accurate pulsar distance, a high pulsar mass and a limit on the variation of Newton's gravitational constant. Astrophys. J. 679, 675-680 (2008)

76. Voges, W., Aschenbach, B., Boller, T., Braeuninger, H., Briel, U., Burkert, W., Dennerl, K., Englhauser, J., Gruber, R., Haberl, F., Hartner, G., Hasinger, G., Pfeffermann, E., Pietsch, W., Predehl, P., Rosso, C., Schmitt, J., Truemper, J., Zimmermann, H.: The ROSAT all-sky survey bright source catalogue. Astron. Astrophys. 349, 389-405 (1999)

77. Voges, W., Aschenbach, B., Boller, T., Brauninger, H., Briel, U., Burkert, W., Dennerl, K., Englhauser, J., Gruber, R., Haberl, F., Hartner, G., Hasinger, G., Pfeffermann, E., Pietsch, W., Predehl, P., Schmitt, J., Trumper, J., Zimmermann, U.: ROSAT All-Sky survey faint source catalog. IAU Circ. 7432, 1 (2000)

78. Watson, M.G., Schroeder, A.C., Fyfe, D., Page, C.G., Lamer, G., Mateos, S., Pye, J., Sakano, M., Rosen, S., Ballet, J., Barcons, X., Barret, D., Boller, T., Brunner, H., Brusa, M., Caccianiga, A., Carrera, F.J., Ceballos, M., Della Ceca, R., Denby, M., Denkinson, G., Dupuy, S., Farrell, S., Fraschetti, F., Freyberg, M.J., Guillout, P., Hambaryan, V., Maccacaro, T., Mathiesen, B., McMahon, R., Michel, L., Motch, C., Osborne, J.P., Page, M., Pakull, M.W., Pietsch, W., Saxton, R., Schwope, A., Severgnini, P., Simpson, M., Sironi, G., Stewart, G., Stewart, I.M., Stobbart, A., Tedds, J., Warwick, R., Webb, N., West, R., Worrall, D.Y.: The XMM-newton serendipitous survey. V. The second XMM-newton serendipitous source catalogue. Astron. Astrophys. 493, 339-373 (2009)

79. Webb, N.A., Olive, J.-F., Barret, D., Kramer, M., Cognard, I., Löhmer, O.: XMM-newton spectral and timing analysis of the faint millisecond pulsars PSR J0751+1807 and PSR J1012+5307. Astron. Astrophys. 419, 269 (2004)

80. Weisberg, J.M., Taylor, J.H.: Observations of post-Newtonian timing effects in the binary pulsar PSR 1913 + 16. Phys. Rev. Lett. 52, 1348-1350 (1984)

81. Willingale, R., Fraser, G.W., Brunton, A.N., Martin, A.P.: Hard X-ray imaging with microchannel plate optics. Exp. Astron. 8, 281-296 (1998)

82. Zane, S., Walton, D., Kennedy, T., et.al.: The large area detector of LOFT: the Large Observatory for Xray Timing. In: Proceedings of Soc. Photo-Opt. Instru. 9144, Space Telescopes and Instrumentation, Quebec, Canada, id. 91442 W, 22 June (2014)

83. Zavlin, V.E.: XMM-Newton observations of four millisecond pulsars. Astrophys. J. 638, 951-962 (2006)

84. Zavlin, V.E.: First X-Ray observations of the young pulsar J1357-6429. Astrophys. J. 665, L143-L146 (2007) 